\title{
A Three-Dimensional MHD Simulation of Plasma Flow Past Io
}

\author{
J. A. LINKER \\ Department of Physics, University of California, Irvine
}

\author{
M. G. KIVELSON ${ }^{1}$ AND R. J. WALKER
}

Institute of Geophysics and Planetary Physics, University of California, Los Angeles

\begin{abstract}
We describe a time-dependent three-dimensional magnetohydrodynamic (MHD) simulation of plasma flow past Jupiter's satellite Io. The simulation starts with a uniform, magnetized flow interacting with a finite conducting sphere (representing Io), and the integration is carried forward in time until a quasi-steady state develops. Previously we have reported on the presence of standing slow mode perturbations in the flow, attached to Io. In this paper we present details of the simulation model and describe the overall features of the interaction. Field-aligned currents are found in regions extending downstream from the obstacle at an angle to the background field; these currents form the Alfvén wings, an expected result. The plasma flow diverts both around Io and the Alfven wings. An "Alfvén ellipse," the intersection of a cylindrical Alfvén wing with an arbitrary plane above Io, describes the obstacle that an Alfvén wing presents to the flow. In addition to the Alfvén mode, which carries the field-aligned currents, perturbations carried by the other MHD modes are also present. Standing slow mode perturbations are the most significant of these, but a fast mode signature also appears. Near Io the interaction is clearly nonlinear; all three MHD modes interact, and the local Alfven and sound speeds are altered. However, estimates of the spatial location of the standing MHD mode disturbances based on the background characteristic velocities are qualitatively accurate.
\end{abstract}

\section{INTRODUCTION}

Io is the innermost of Jupiter's four Galilean satellites. The first indication of Io's importance in the Jovian magnetosphere came from the discovery by Bigg [1964] that Jovian radio emissions are controlled by Io's position. Since that time, further observations have confirmed that Io has unusual properties. The first spacecraft to fly through the Jovian magnetosphere was Pioneer 10. Although no in situ measurements were made near Io, the Pioneer 10 radio occultation experiment found evidence for an ionosphere surrounding Io [Kliore et al., 1974]. Ground-based observations of sodium near Io [Brown, 1974; Trafton et al., 1974; Mekler and Eviatar, 1974] revealed that Io is a source of neutral particles. Subsequent observations have shown that clouds of neutral potassium, oxygen, and sulfur are present near Io [Brown and Ip, 1981; Pilcher and Strobel, 1982; Durrance et al., 1983]. The detection of $\mathrm{S}^{+}$emissions inside Io's orbit [Kupo et al., 1976] gave the first evidence linking a heavy ion plasma with Io.

The most spectacular discoveries regarding lo awaited the Voyager 1 flyby in 1979 . Voyager images revealed that Io is the most volcanically active body known in the solar system [Strom et al., 1981]. Passing within $20,000 \mathrm{~km}$ (about 11 Io radii) of Io, Voyager 1 made the first in situ measurements of plasma near Io and found large field-aligned currents flowing along the Io flux tube, the first direct evidence of a strong interac-

\footnotetext{
${ }^{1}$ Also at Department of Earth and Space Science, University of California, Los Angeles.

Copyright 1991 by the American Geophysical Union.

Paper number 91JA02132.

0148-0227/91/91 JA-02132\$05.00
}

tion between Io and the Jovian magnetic field [Ness et al., 1979; Acuna et al., 1981]. Voyager 1 found a dense plasma localized in a torus about Jupiter centered near Io's orbit, with a minor axis diameter of about 2 Jovian radii. This feature, dominated by sulfur and oxygen ions [Broadfoot et al., 1979; Bagenal and Sullivan, 1981], has since been referred to as the Io plasma torus.

The field-aligned current associated with Io was not unexpected. Io's control of the Jovian radio emissions led quite early to the idea that plasma near Io is coupled electrodynamically with the Jovian ionosphere. The coupling of two remote plasmas along magnetic field lines implies the generation of field-aligned currents. The MHD wave mode that carries field-aligned currents is the Alfvén wave. It was Drell et al. [1965], in studying drag effects on satellites in the Earth's ionosphere, who first described the Alfven perturbation that stands in the rest frame of a conductor embedded in a flowing plasma. For the Alfvén wave in a uniform plasma the group velocity is magnetic field guided in the plasma rest frame. In the conductor's rest frame the Alfvén perturbation is swept back by the background flow. The leading edge of the perturbation region lies along the Alfven characteristic, at an angle $\tan ^{-1} M_{A}$ to the background magnetic field, where $M_{A}$ is the Alfvén Mach number. This region, where the perturbation currents flow, is known as the Alfvén wing. Marshall and Libby [1967] linked the idea of a standing Alfvén wave at Io to Io's control of the radio emissions.

Much of the early work on the generation of fieldaligned currents caused by Io's perturbation of the Jovian magnetic field assumed that the Jovian and Ionian ionospheres were directly linked by the currents. Estimates for the round-trip travel time for an Alfvén wave generated at Io, reflected at the Jovian ionosphere, and 
propagating back to lo were less than $1 \mathrm{~min}$, a time short enough for the Alfven wave to return to Io before the local plasma could convect past lo. In these "unipolar" models [Goldreich and Lynden-Bell, 1969; Goertz and Deifl, 1973], the Ionian and Jovian ionospheric currents form part of a closed circuit, and the conductivity of the Jovian ionosphere directly controls the current flow at Io.

The Voyager 1 flyby confirmed the presence of the field-aligned currents, but the unexpectedly high density and low Alfvén speed of the torus plasma implied a much longer $(\approx 20 \mathrm{~min})$ round-trip travel time for Alfvén waves between the Ionian and Jovian ionospheres. For this much longer travel time, Io has moved significantly from its previous position (in the plasma rest frame) by the time the reflected Alfven wave arrives, and the circuit cannot close locally; the currents must close elsewhere in the torus. From the point of view of Io's rest frame, the reflected Alfven wave returns downstream of Io. When Io is near the edge of the plasma torus, a direct interaction could still occur [Neubauer, 1980]; however, when Io is located deeper within the torus, the flow of the plasma past Io produces a "local" interaction, i.e., one in which the perturbations produced at Io are purely radiative, and the Jovian ionosphere does not directly influence the flow in the vicinity of Io. Models developed after the discovery of the torus plasma have concentrated on the local interaction, as we do in our simulations.

In describing the local Alfven wave interaction at Io, many authors [e.g., Neubauer, 1980; Southwood et al., 1980; Southwood and Dunlop, 1984] have characterized the current in the Alfven waves by the Alfven conductance $\left(\Sigma_{A} \approx 1 /\left(\mu_{0} V_{A}\right)\right.$, in MKS units where $V_{A}$ is the Alfvén velocity). For the local interaction, the current generated at lo must be carried off by the Alfven wings, which requires

$$
\Sigma_{\mathrm{Io}} E_{\mathrm{Io}}=2 \Sigma_{A}\left(E_{u}-E_{\mathrm{Io}}\right)
$$

Here $\Sigma_{\mathrm{Io}}$ is Io's conductance, $E_{\mathrm{Io}}$ is the electric field across Io, and $E_{u}$ is the undisturbed (background flow) electric field. The factor of 2 arises because current flows away from Io toward both north and south. If we parameterize $E_{\mathrm{Io}}$ as $\alpha E_{u}$, the flow electric field at Io is reduced by the factor $\alpha$ [Southwood et al, 1980, Southwood and Dunlop, 1984].

$$
\alpha=\frac{2 \Sigma_{A}}{\Sigma_{\mathrm{Io}}+2 \Sigma_{A}}=\frac{1}{1+\mu_{0} V_{A} \Sigma_{\mathrm{Io}} / 2}
$$

If the Alfvén conductance is much higher than Io's conductance, the flow electric field at Io is largely unaffected. If Io's conductance is much greater than the Alfvén conductance, the flow electric field is nearly shorted out at Io. Io's conductivity brings the flow to a halt.

Detailed models of the standing Alfvén wave pattern near Io have been derived. Neubauer [1980] extended the work of Drell et al. [1965] and developed a nonlinear, analytic solution for the Alfvén wings far away from Io. Wright and Southwood [1987] examined the topology and closure of the perpendicular current system and its relation to the field-aligned currents.

While these papers describe the Alfvén perturbations far from Io, they do not model compressional perturbations or the flow close to Io. The first paper to provide a nonlinear description of the interaction near lo was that of Wolf-Gladrow et al. [1987]. They modeled numerically the magnetic field and current perturbations close to Io self-consistently, and they examined the effects that different Io ionospheric models have on the resulting currents near lo. They found that models with a wide range of ionospheric conductivities (54-126 mhos) matched the Voyager 1 observations reasonably well, in part because Voyager passed upstream of the Alfven wing and not directly through it, so the measurements did not probe regions directly influenced by the ionosphere. Their calculations ignored pressure gradients and flow parallel to the magnetic field, so effects such as those produced by slow mode waves were: not included.

In this paper we focus on the flow of the torus plasma in the immediate neighborhood of Io using three-dimensional MHD simulations. Such simulations have been used previously to model the interaction of the solar wind with the Earth's magnetosphere [e.g., Leboeuf et al., 1981; Wu et al., 1981; Brecht et al., 1982; Ogino, 1986; Fedder and Lyon, 1987] and with comets [e.g., Schmidt and Wegmann, 1982; Ogino et al., 1988] for the case of supermagnetosonic flow. The primary goal of our study is to examine effects on the plasma flow near Io in the transonic and submagnetosonic regime. We obtain quasi-steady configurations of the plasma and magnetic field (by quasi-steady we mean that there may be small fluctuations in the numerical values of various quantities, but the qualitative features we describe are no longer changing). As we solve the three-dimensional, time-dependent, compressible MHD equations, perturbations to the flow can be produced by all three of the MHD wave modes. We have found that in addition to the Alfvén mode, other MHD modes can contribute important perturbations, particularly the slow mode (recently, Wright and Schwartz [1990] have also argued that slow mode waves should be important near Io). Some of our principal results from these simulations (e.g., effects of the slow mode and of mass loading) have been reported elsewhere [Linker et al., 1988; Linker et al., 1989]. Here we provide details of the simulation method (in particular, the solution of the MHD equations in spherical coordinates) as well as a description of the general features of the interaction. We describe the plasma properties including density, pressure, and flow velocity near Io and the Alfvén wing, and we identify the magnetic field topology and the patterns of current flow in and near the Alfvén wing. We also show the nonlinear effects of slow and fast mode perturbations on the plasma properties, and the resultant changes that occur in the Alfvén and sound velocities near Io.

In this paper we do not consider the possibility that Io has an intrinsic magnetic field, although it is not ruled out by observations [Neubauer, 1978; Kivelson et al., 1979; Southwood et al., 1980]. The lack of a complete dropout in the flux of very energetic $(\geq 10 \mathrm{MeV})$ electrons gives possible evidence for an intrinsic magnetic field. The mirroring of electrons with large pitch angles at Io's polar cap in the presence of an intrinsic field could explain the survival of the energetic electrons. Wright [1987] has also advanced an explanation for this effect that does not require an intrinsic field at Io. 


\section{Description of the Simulation}

We solve the following form of the compressible MHD equations as an initial value problem:

$$
\begin{gathered}
\frac{\partial \rho}{\partial t}+\nabla \cdot(\rho \vec{v})=0 \\
\frac{d}{d t}\left(P \rho^{-\gamma}\right)=\frac{\gamma-1}{\rho^{\gamma}}\left(\eta J^{2}-\frac{1}{R_{e}} \mathbf{W}: \nabla \vec{v}\right) \\
\frac{\partial}{\partial t}(\rho \vec{v})+\nabla \cdot\left(\rho \vec{v} \vec{v}+\left(P+\frac{B^{2}}{2}\right) \mathbf{I}-\vec{B} \vec{B}\right) \\
=-\frac{1}{R_{e}} \nabla \cdot \mathbf{W} \\
\frac{\partial \vec{B}}{\partial t}-\nabla \times(\vec{v} \times \vec{B})=-\nabla \times(\eta \vec{J})
\end{gathered}
$$

In the above equations, $\rho$ is the density, $\vec{v}$ the velocity, $P$ the plasma pressure (assumed to be isotropic), $\vec{B}$ the magnetic field, and $\vec{J}=\nabla \times \vec{B}$ the current density. W is a viscous stress tensor used to main tain numerical stabil-

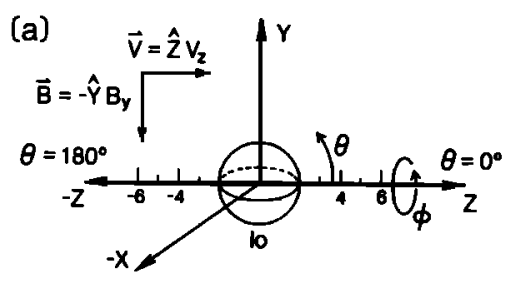

(b)

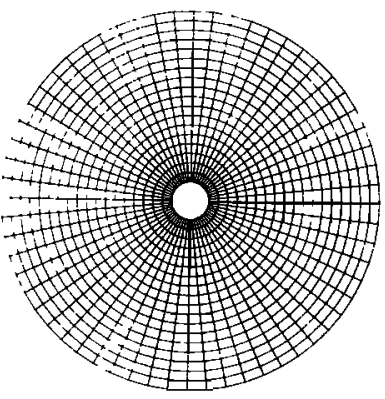

(c)

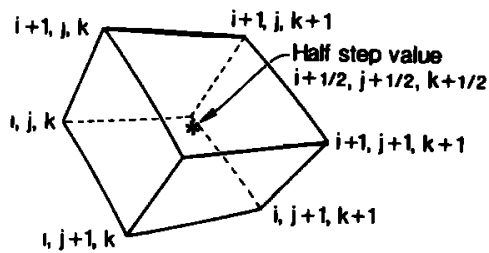

Fig. 1. (a) The coordinate system used in the simulation; $\theta=180^{\circ}$ is upstream of Io; $\theta=0^{\circ}$ is downstream of Io. (b) A two-dimensional "slice" of the simulation grid, e.g., for the xy plane $\left(\phi=0^{\circ}\right.$ and $\left.\phi=180^{\circ}\right)$. Io's surface is two radial cells from the center $\left(\Delta r=0.5 R_{\mathrm{Io}}, \Delta \theta=6^{\circ}\right.$, $\left.\Delta \phi=10^{\circ}\right)$. (c) A typical cell in the simulation grid. The first half step is calculated as a Lax step; the second half step is a leapfrog step. Both steps use explicit temporal differencing and centered eight-point spatial differencing. ity. $R_{e}$ is the fluid Reynolds number, $\eta$ is the resistivity, and $\gamma=5 / 3$. The equations are given in normalized units, so, for example, $\rho=\rho^{\prime} / \rho_{0}$, and $\vec{v}=\vec{v}^{\prime} / v_{0}$, where $\rho^{\prime}$ and $\vec{v}^{\prime}$ are the density and velocity in cgs units and $\rho_{0}$ and $v_{0}$ are typical values of the torus density and velocity. Similar definitions are used for all the simulation variables; in these units, typical MHD quantities such as the Alfvén speed $\left(V_{A}=B / \sqrt{\rho}\right)$, magnetic pressure $\left(B^{2} / 2\right.$ ), and $\beta$ (ratio of plasma to magnetic pressure, $2 P / B^{2}$ ) appear without factors of $4 \pi$ or $\mu_{0}$. The length scale $L$ used for normalization is $1 / 2 R_{\mathrm{Io}}$ (Io radius).

The equations are solved in spherical coordinates by using a two-step Lax-Wendroff method. Figure $1 a$ shows the simulation coordinate system; Figure $1 b$ shows a projection of the simulation grid on the $y z$ plane (equivalent to the $\phi=90^{\circ}$ and $\phi=270^{\circ}$ planes). Although a grid defined in spherical coordinates introduces some computational complexity, it has a number of advantages for studying flow past planetlike obstacles such as Io. The center of Io is located at the center of the spherical coordinate system, so that high resolution near Io is obtained without the expense of high resolution everywhere in the simulation. Defining a spherical boundary for Io is easily accomplished. However, solving the MHD equations in spherical coordinates requires some alteration of the usual implementation of finite difference algorithms such as Lax-Wendroff. We describe these techniques in the following section.

\subsection{Solving the MHD Equations in Spherical Coordinates}

Equations (2)-(5) can be expressed in spherical coordinates (away from $(\theta=0, \pi)$ ) as

Mass equation

$$
\begin{gathered}
\frac{\partial \rho}{\partial t}+\frac{1}{r^{2}} \frac{\partial}{\partial r}\left(r^{2} \rho v_{r}\right)+\frac{1}{r \sin \theta} \frac{\partial}{\partial \theta}\left(\rho v_{\theta} \sin \theta\right) \\
+\frac{1}{r \sin \theta} \frac{\partial}{\partial \phi}\left(\rho v_{\phi}\right)=0
\end{gathered}
$$

Equation for $P \rho^{-\gamma}$

$$
\begin{gathered}
\frac{\partial}{\partial t}\left(P \rho^{-\gamma}\right)+v_{r} \frac{\partial}{\partial r}\left(P \rho^{-\gamma}\right)+\frac{v_{\theta}}{r} \frac{\partial}{\partial \theta}\left(P \rho^{-\gamma}\right) \\
+\frac{v_{\phi}}{r \sin \theta} \frac{\partial}{\partial \phi}\left(P \rho^{-\gamma}\right) \\
=\frac{\gamma-1}{\rho^{\gamma}}\left(\eta\left(J_{r}^{2}+J_{\theta}^{2}+J_{\theta}^{2}\right)-\frac{1}{R_{e}} \mathbf{W}: \nabla \vec{v}\right)
\end{gathered}
$$

Components of the momentum equation

$$
\begin{gathered}
\frac{\partial}{\partial t}\left(\rho v_{r}\right)+\frac{1}{r^{2}} \frac{\partial}{\partial r}\left(r^{2}\left(\rho v_{r}^{2}+P+\frac{B_{\theta}^{2}+B_{\phi}^{2}-B_{r}^{2}}{2}\right)\right) \\
+\frac{1}{r \sin \theta} \frac{\partial}{\partial \theta}\left(\sin \theta\left(\rho v_{\theta} v_{r}-B_{\theta} B_{r}\right)\right) \\
\quad+\frac{1}{r \sin \theta} \frac{\partial}{\partial \phi}\left(\rho v_{\phi} v_{r}-B_{\phi} B_{r}\right) \\
=\frac{\left(\rho v_{\theta}^{2}+2 P+\rho v_{\phi}^{2}+B_{r}^{2}\right)}{r}-\frac{1}{R_{e}}(\nabla \cdot \mathrm{W})_{r}
\end{gathered}
$$




$$
\begin{gathered}
\frac{\partial}{\partial t}\left(\rho v_{\theta}\right)+\frac{1}{r^{2}} \frac{\partial}{\partial r}\left(r^{2}\left(\rho v_{r} v_{\theta}-B_{r} B_{\theta}\right)\right) \\
+\frac{1}{r \sin \theta} \frac{\partial}{\partial \theta}\left(\sin \theta\left(\rho v_{\theta}^{2}+P+\frac{B_{r}^{2}+B_{\phi}^{2}-B_{\theta}^{2}}{2}\right)\right) \\
+\frac{1}{r \sin \theta} \frac{\partial}{\partial \phi}\left(\rho v_{\phi} v_{\theta}-B_{\phi} B_{\theta}\right) \\
=\frac{-\rho v_{\theta} v_{r}+B_{\theta} B_{r}}{r} \\
+\frac{\cos \theta}{r \sin \theta}\left(\rho v_{\phi}^{2}+P+\frac{B_{r}^{2}+B_{\theta}^{2}-B_{\phi}^{2}}{2}\right) \\
-\frac{1}{R_{e}}(\nabla \cdot \mathrm{W})_{\theta} \\
\frac{\partial}{\partial t}\left(\rho v_{\phi}\right)+\frac{1}{r^{2}} \frac{\partial}{\partial r}\left(r^{2}\left(\rho v_{r} v_{\phi}-B_{r} B_{\phi}\right)\right) \\
+\frac{1}{r \sin \theta} \frac{\partial}{\partial \theta}\left(\sin \theta\left(\rho v_{\theta} v_{\phi}-B_{\theta} B_{\phi}\right)\right) \\
+\frac{1}{r \sin \theta} \frac{\partial}{\partial \phi}\left(\rho v_{\phi}^{2}+P+\frac{B_{r}^{2}+B_{\theta}^{2}-B_{\phi}^{2}}{2}\right) \\
=\frac{-\rho v_{\phi} v_{r}+B_{\phi} B_{r}}{r}-\frac{\cos \theta}{r \sin \theta}\left(\rho v_{\phi} v_{\theta}-B_{\phi} B_{\theta}\right) \\
-\frac{1}{R_{e}}(\nabla \cdot \mathrm{W})_{\phi}
\end{gathered}
$$

Components of Faraday's law

$$
\begin{gathered}
\frac{\partial B_{r}}{\partial t}+\frac{1}{r^{2} \sin \theta} \frac{\partial}{\partial \theta}\left(r \sin \theta\left(v_{\theta} B_{r}-v_{r} B_{\theta}\right)\right) \\
+\frac{1}{r^{2} \sin \theta} \frac{\partial}{\partial \phi} r\left(v_{\phi} B_{r}-v_{r} B_{\phi}\right) \\
=\frac{1}{r^{2} \sin \theta} \frac{\partial}{\partial \theta}\left(-r \sin \theta \eta J_{\phi}\right)+\frac{1}{r^{2} \sin \theta} \frac{\partial}{\partial \phi}\left(r \eta J_{\theta}\right) \\
\frac{\partial B_{\theta}}{\partial t}+\frac{1}{r \sin \theta} \frac{\partial}{\partial r}\left(r \sin \theta\left(v_{r} B_{\theta}-v_{\theta} B_{r}\right)\right) \\
+\frac{1}{r \sin \theta} \frac{\partial}{\partial \phi}\left(v_{\phi} B_{\theta}-v_{\theta} B_{\phi}\right) \\
\frac{1}{r \sin \theta} \frac{\partial}{\partial r}\left(r \sin \theta \eta J_{\phi}\right)+\frac{1}{r \sin \theta} \frac{\partial}{\partial \phi}\left(-\eta J_{r}\right) \\
\frac{\partial B_{\phi}}{\partial t}+\frac{1}{r} \frac{\partial}{\partial r}\left(r\left(v_{r} B_{\phi}-v_{\phi} B_{r}\right)\right) \\
+\frac{1}{r} \frac{\partial}{\partial \theta}\left(v_{\theta} B_{\phi}-v_{\phi} B_{\theta}\right) \\
=\frac{1}{r} \frac{\partial}{\partial r}\left(-r \eta J_{\theta}\right)+\frac{1}{r} \frac{\partial}{\partial \theta}\left(\eta J_{r}\right)
\end{gathered}
$$

The current density vectors are defined by the equations

$$
\begin{gathered}
J_{r}=\frac{1}{r^{2} \sin \theta}\left(\frac{\partial}{\partial \theta}\left(r \sin \theta B_{\phi}\right)-\frac{\partial r B_{\theta}}{\partial \phi}\right) \\
J_{\theta}=\frac{1}{r \sin \theta} \frac{\partial B_{r}}{\partial \phi}-\frac{1}{r \sin \theta} \frac{\partial}{\partial r}\left(r \sin \theta B_{\phi}\right) \\
J_{\phi}=\frac{1}{r} \frac{\partial}{\partial r}\left(r B_{\theta}\right)-\frac{1}{r} \frac{\partial B_{r}}{\partial \theta}
\end{gathered}
$$

For simplicity we have left the viscous terms in an unexpanded form. Equations (6)-(16) are solved by using the modified two-step Lax-Wendroff method [e.g, Potter, 1973; Ogino, 1986] in much the same way as in Cartesian coordinates. Figure $1 c$ shows a typical cell in the simulation grid. The first half step is calculated as a Lax step; the second half step is a leapfrog step. Both steps use explicit temporal differencing and centered eight-point spatial differencing. Nondissipative "source" terms appearing in the equations, such as the term $\left(\rho v_{\theta}^{2}+2 P+\rho v_{\phi}^{2}+B_{r}^{2}\right) / r$ in (8) are included in the half step calculation by averaging the neighboring full-step values. At the second half step, values at the previous (full) time step are used to calculate the source terms. The algorithm has second-order accuracy.

We note here that the equations for Faraday's law, (11)-(13), are solved in the form shown, rather than by expanding the derivatives. The reason for this approach is that it allows us to maintain $(\partial / \partial t) \nabla \cdot \vec{B}=0$ to round-off error, as opposed to truncation error. For finite-difference calculations on even the smallest meshes possible, the truncation errors of an algorithm (due to the inaccuracy of the scheme, typically $10^{-4}$ to $10^{-6}$ for 1 step) are generally 8-10 orders of magnitude greater than round-off errors caused by finite machine precision (for 64-bit computations). If we express the variations of $\vec{B}$ by using an equation in the form of Faraday's law, then the perturbation to $\vec{B},(\delta \vec{B})$, calculated at each time step is equal to the curl of the electric field. We formulate the divergence and curl operators such that the analytic vector identity $\nabla \cdot \nabla \times=0$ is preserved to round-off error. (In this regard note that we incorporate the full metric in performing the finite differencing in equations (11)-(13.) Then $\nabla \cdot \delta \vec{B}=0$ to round-off error, so $(\partial / \partial t) \nabla \cdot \vec{B}=0$ to round-off error.

Dissipative source terms, such as the viscous terms in (8)-(10), were calculated by using first-order explicit differencing. Resistive terms were calculated by formulating the current density at half-step values and then taking the curl of these values to obtain the full-step value, again ensuring that the change in $\bar{B}$ is calculated as the curl of the electric field. The resistive and dissipative terms (kept as small as possible) are included to help damp out short-wavelength ripples generated by numerical dispersion, while leaving the longer-wavelength phenomena minimally affected. For the results shown in this paper, $R_{e}=50$, and $S$, the magnetic Lundquist number $\left(V_{A} L / \eta\right.$, where $V_{A}=B / \sqrt{\rho}$ is the Alfvén speed and the length scale $L$ is an lo diameter) is 100 . We have performed simulations with $R_{e}$ and $S$ in the range of 10-200. The results remain qualitatively unchanged in this range, but higher levels of numerical fluctuations occur in the higher Reynolds number cases.

We must specify how to obtain a solution at the "poles" of the spherical coordinate system, since (6)(16) are not defined at $\theta=0, \pi$. The approach that we take is to derive a new set of equations valid only at $\theta=0, \pi$, as has been done previously for the gasdynamic equations [e.g, Bohachevsky and Mates, 1966]. The procedure is straightforward but sufficiently complicated that it is worth writing out the results for the full MHD equations. Note that in spherical coordi- 
nates, as $\theta \rightarrow 0, \pi$, the quantities $\sin \theta,(\partial / \partial \phi) \rightarrow 0$. For (6)-(16), all of the terms that contain the singularities (terms of the form $(1 / r \sin \theta)(\partial / \partial \theta) \sin \theta()$ or $(1 / r \sin \theta)(\partial / \partial \phi)())$ approach zero in the numerator as well as in the denominator. We derive a new set of equations valid for $\lim (\theta \rightarrow 0, \pi,(\partial / \partial \phi) \rightarrow 0)$ from $(6-$ 16) by using L'Hôpital's rule. As an example, consider the last two terms in the mass equation (6):

$$
\begin{aligned}
& \lim (\theta \rightarrow 0, \pi) {\left[\frac{1}{r \sin \theta} \frac{\partial}{\partial \theta}\left(\rho v_{\theta} \sin \theta\right)\right] } \\
&=\left[\frac{1}{r \cos \theta} \frac{\partial^{2}}{\partial \theta^{2}}\left(\rho v_{\theta} \sin \theta\right)\right]_{\theta=0, \pi} \\
&=\frac{2}{r} \frac{\partial}{\partial \theta}\left(\rho v_{\theta}\right) \\
& \lim \left(\theta \rightarrow 0, \pi, \frac{\partial}{\partial \phi} \rightarrow 0\right) {\left[\frac{1}{r \sin \theta} \frac{\partial}{\partial \phi}\left(\rho v_{\phi}\right)\right] } \\
&= {\left[\frac{1}{r \cos \theta} \frac{\partial^{2}}{\partial \theta \partial \phi}\left(\rho v_{\phi}\right)\right]_{\theta=0, \pi} } \\
&= \pm \frac{1}{r} \frac{\partial^{2}}{\partial \theta \partial \phi}\left(\rho v_{\phi}\right)
\end{aligned}
$$

where the upper sign is at $\theta=0$ and the lower sign is at $\theta=\pi$, a convention to which we will adhere in all subsequent equations and discussion. The full mass equation at the poles becomes

$$
\frac{\partial \rho}{\partial t}+\frac{1}{r^{2}} \frac{\partial}{\partial r}\left(r^{2} \rho v_{r}\right)+\frac{2}{r} \frac{\partial}{\partial \theta}\left(\rho v_{\theta}\right) \pm \frac{1}{r} \frac{\partial^{2}}{\partial \theta \partial \phi}\left(\rho v_{\phi}\right)=0
$$

Using the same procedure, (7)-(13) become

$$
\begin{gathered}
\frac{\partial}{\partial t}\left(P \rho^{-\gamma}\right)+v_{r} \frac{\partial}{\partial r}\left(P \rho^{-\gamma}\right)+\frac{v_{\theta}}{r} \frac{\partial}{\partial \theta}\left(P \rho^{-\gamma}\right) \\
\pm \frac{v_{\phi}}{r} \frac{\partial^{2}}{\partial \theta \partial \phi}\left(P \rho^{-\gamma}\right) \\
=\frac{\gamma-1}{\rho^{\gamma}}\left(\eta\left(J_{r}^{2}+J_{\theta}^{2}+J_{\theta}^{2}\right)-\frac{1}{R_{e}} \mathrm{~W}: \nabla \vec{v}\right) \\
\frac{\partial}{\partial t}\left(\rho v_{r}\right)+\frac{1}{r^{2}} \frac{\partial}{\partial r}\left(r^{2}\left(\rho v_{r}^{2}+P+\frac{B_{\theta}^{2}+B_{\phi}^{2}-B_{r}^{2}}{2}\right)\right) \\
+\frac{2}{r} \frac{\partial}{\partial \theta}\left(\rho v_{\theta} v_{r}-B_{\theta} B_{r}\right) \pm \frac{1}{r} \frac{\partial^{2}}{\partial \theta \partial \phi}\left(\rho v_{\phi} v_{r}-B_{\phi} B_{r}\right) \\
=\frac{\left(\rho v_{\theta}^{2}+2 P+\rho v_{\phi}^{2}+B_{r}^{2}\right)}{r}-\frac{1}{R_{e}}(\nabla \cdot \mathrm{W})_{r} \\
\frac{\partial}{\partial t}\left(\rho v_{\theta}\right)+\frac{1}{r^{2}} \frac{\partial}{\partial r}\left(r^{2}\left(\rho v_{r} v_{\theta}-B_{r} B_{\theta}\right)\right) \\
+\frac{1}{r} \frac{\partial}{\partial \theta}\left(2 \rho v_{\theta}^{2}-\rho v_{\phi}^{2}+P+\frac{B_{\tau}^{2}+B_{\phi}^{2}-B_{\theta}^{2}}{2}\right) \\
\pm \frac{1}{r} \frac{\partial^{2}}{\partial \theta \partial \phi}\left(\rho v_{\phi} v_{\theta}-B_{\phi} B_{\theta}\right) \\
=\frac{-\rho v_{\theta} v_{r}+B_{\theta} B_{r}}{r}-\frac{1}{R_{e}}(\nabla \cdot \mathrm{W})_{\theta}
\end{gathered}
$$

$$
\begin{aligned}
& \frac{\partial}{\partial t}\left(\rho v_{\phi}\right)+\frac{1}{r^{2}} \frac{\partial}{\partial r}\left(r^{2}\left(\rho v_{r} v_{\phi}-B_{r} B_{\phi}\right)\right) \\
& +\frac{3}{r} \frac{\partial}{\partial \theta}\left(\rho v_{\theta} v_{\phi}-B_{\theta} B_{\phi}\right) \\
& \pm \frac{1}{r} \frac{\partial^{2}}{\partial \theta \partial \phi}\left(\rho v_{\phi}^{2}+P+\frac{B_{r}^{2}+B_{\theta}^{2}-B_{\phi}^{2}}{2}\right) \\
& =\frac{-\rho v_{\phi} v_{r}+B_{\phi} B_{r}}{r}-\frac{1}{R_{e}}(\nabla \cdot \mathrm{W})_{\phi} \\
& \frac{\partial B_{r}}{\partial t}+\frac{2}{r^{2}} \frac{\partial}{\partial \theta}\left(r\left(v_{\theta} B_{r}-v_{r} B_{\theta}\right)\right) \\
& \pm \frac{1}{r^{2}} \frac{\partial^{2}}{\partial \theta \partial \phi} r\left(v_{\phi} B_{r}-v_{r} B_{\phi}\right) \\
& =\frac{2}{r^{2}} \frac{\partial}{\partial \theta}\left(-r \eta J_{\phi}\right) \pm \frac{1}{r} \frac{\partial^{2}}{\partial \theta \partial \phi}\left(r \eta J_{\theta}\right) \\
& \frac{\partial B_{\theta}}{\partial t}+\frac{1}{r} \frac{\partial}{\partial r}\left(r\left(v_{r} B_{\theta}-v_{\theta} B_{r}\right)\right) \\
& \pm \frac{1}{r} \frac{\partial^{2}}{\partial \theta \partial \phi}\left(v_{\phi} B_{\theta}-v_{\theta} B_{\phi}\right) \\
& =\frac{1}{r} \frac{\partial}{\partial r}\left(r \eta J_{\phi}\right) \pm \frac{1}{r} \frac{\partial^{2}}{\partial \theta \partial \phi}\left(-\eta J_{r}\right) \\
& \frac{\partial B_{\phi}}{\partial t}+\frac{1}{r} \frac{\partial}{\partial r}\left(r\left(v_{r} B_{\phi}-v_{\phi} B_{r}\right)\right) \\
& +\frac{1}{r} \frac{\partial}{\partial \theta}\left(v_{\theta} B_{\phi}-v_{\phi} B_{\theta}\right) \\
& =\frac{1}{r} \frac{\partial}{\partial r}\left(-r \eta J_{\theta}\right)+\frac{1}{r} \frac{\partial}{\partial \theta}\left(\eta J_{r}\right)
\end{aligned}
$$

We do not evaluate the limits of (14)-(16) for the current density. This is because the current density is calculated only at the half step, so limits valid at the poles are not needed (the $\eta J^{2}$ term in (18) is determined by averaging the half step values).

Given a set of MHD equations valid at $\theta=0, \pi$ (in addition to the usual spherical coordinate equations valid away from the poles), we still must specify how the finite difference algorithm is to be applied to these equations. To apply the Lax-Wendroff algorithm at the pole, the pole values must be calculated from the half-step values calculated nearest the pole. One problem that arises in attempting a finite-difference calculation at the pole is that because of the convergence of the coordinate system at the poles, there are more half-step values around the pole than are necessary to perform the usual eightpoint centered difference. In our algorithm we chose to calculate the pole values with an eight-point difference by using a subset of the half-step values next to the pole, specifically the $\phi$ values of $45^{\circ}, 135^{\circ}, 225^{\circ}$, and $315^{\circ}$ (our choice of the mesh in the $\phi$ direction is made such that these $\phi$ values always correspond to half-step locations). The pole value at a given $r_{i}$ is then determined by using the four values at $r_{i-1 / 2}, \phi=45^{\circ}$, 
$\phi=135^{\circ}, \phi=225^{\circ}, \phi=315^{\circ}$ and the four values at $r_{i+1 / 2}, \phi=45^{\circ}, \phi=135^{\circ}, \phi=225^{\circ}, \phi=315^{\circ}$, all at $\Delta \theta / 2$ away from the pole.

To solve the equations near the poles, we must approximate the derivatives $(\partial / \partial \theta)$ and $\left(\partial^{2} / \partial \theta \partial \phi\right)$ using the eight half-step values next to the pole. The appropriate way to approximate these derivatives at the pole by using finite differences is not obvious. One way to correctly interpret these derivatives is to express them in a local Cartesian coordinate system. Using

$$
\begin{aligned}
& x=r \sin \theta \cos \phi \\
& y=r \sin \theta \sin \phi \\
& z=r \cos \theta
\end{aligned}
$$

and the partial derivative relations

$$
\begin{aligned}
\frac{\partial}{\partial r} & =\frac{\partial x}{\partial r} \frac{\partial}{\partial x}+\frac{\partial y}{\partial r} \frac{\partial}{\partial y}+\frac{\partial z}{\partial r} \frac{\partial}{\partial z} \\
\frac{\partial}{\partial \theta} & =\frac{\partial x}{\partial \theta} \frac{\partial}{\partial x}+\frac{\partial y}{\partial \theta} \frac{\partial}{\partial y}+\frac{\partial z}{\partial \theta} \frac{\partial}{\partial z} \\
\frac{\partial}{\partial \phi} & =\frac{\partial x}{\partial \phi} \frac{\partial}{\partial x}+\frac{\partial y}{\partial \phi} \frac{\partial}{\partial y}+\frac{\partial z}{\partial \phi} \frac{\partial}{\partial z} \\
\frac{\partial^{2}}{\partial \theta \partial \phi} & =\frac{\partial x}{\partial \phi} \frac{\partial^{2}}{\partial \theta \partial x}+\frac{\partial^{2} x}{\partial \theta \partial \phi} \frac{\partial}{\partial x}+\frac{\partial y}{\partial \phi} \frac{\partial^{2}}{\partial \theta \partial y} \\
& +\frac{\partial^{2} y}{\partial \theta \partial \phi} \frac{\partial}{\partial y}+\frac{\partial z}{\partial \phi} \frac{\partial^{2}}{\partial \theta \partial z}+\frac{\partial^{2} z}{\partial \theta \partial \phi} \frac{\partial}{\partial z}
\end{aligned}
$$

we can write

$$
\begin{aligned}
\frac{\partial}{\partial r} & =\sin \theta \cos \phi \frac{\partial}{\partial x}+\sin \theta \sin \phi \frac{\partial}{\partial y}+\cos \theta \frac{\partial}{\partial z} \\
\frac{\partial}{\partial \theta} & =r \cos \theta \cos \phi \frac{\partial}{\partial x}+r \cos \theta \sin \phi \frac{\partial}{\partial y}-r \sin \theta \frac{\partial}{\partial z} \\
\frac{\partial}{\partial \phi} & =-r \sin \theta \sin \phi \frac{\partial}{\partial x}+r \sin \theta \cos \phi \frac{\partial}{\partial y} \\
\frac{\partial^{2}}{\partial \theta \partial \phi} & =-r \sin \theta \sin \phi \frac{\partial^{2}}{\partial \theta \partial x}-r \cos \theta \sin \phi \frac{\partial}{\partial x} \\
& +r \sin \theta \frac{\partial^{2}}{\partial \theta \partial y}+r \cos \theta \cos \phi \frac{\partial}{\partial y}
\end{aligned}
$$

For $\theta \rightarrow 0, \pi$, these become

$$
\begin{aligned}
\frac{\partial}{\partial r} & = \pm \frac{\partial}{\partial z} \\
\frac{1}{r} \frac{\partial}{\partial \theta} & = \pm \cos \phi \frac{\partial}{\partial x} \pm \sin \phi \frac{\partial}{\partial y} \\
\frac{\partial}{\partial \phi} & =0 \\
\frac{1}{r} \frac{\partial^{2}}{\partial \theta \partial \phi} & =\mp \sin \phi \frac{\partial}{\partial x} \pm \cos \phi \frac{\partial}{\partial y}
\end{aligned}
$$

Note that, as one would expect, the radial direction at the poles corresponds to the $z$ direction in Cartesian coordinates, and $(\partial / \partial \phi)=0$ at the poles. Precisely at the poles, the choice of $\phi$ is arbitrary, because all values correspond to the same point in space. However, (25)(28) show that the choice of $\phi$ determines the orientation of the Cartesian coordinate system. For simplicity, we solve first for $\phi=0$. A different choice of $\phi$ is equivalent to a rotation of the Cartesian coordinate system about the $\mathrm{z}$ axis. With $\phi=0$, equations (25)-(28) become

$$
\begin{aligned}
\frac{\partial}{\partial r} & = \pm \frac{\partial}{\partial z} \\
\frac{1}{r} \frac{\partial}{\partial \theta} & = \pm \frac{\partial}{\partial x} \\
\frac{1}{r} \frac{\partial^{2}}{\partial \theta \partial \phi} & = \pm \frac{\partial}{\partial y}
\end{aligned}
$$

Interpretation of $(\partial / \partial \theta)$ and $\left(\partial^{2} / \partial \theta \partial \phi\right)$ at the poles in terms of finite differences is now straightforward. For example, $(\partial / \partial \theta)$ is determined by subtracting the values at $\phi=135^{\circ}, 225^{\circ}$ from the values at $\phi=45^{\circ}, 315^{\circ}$, i.e., evaluating the change in the $x$ direction; $\left(\partial^{2} / \partial \theta \partial \phi\right)$ is determined by subtracting the values at $\phi=225^{\circ}, 315^{\circ}$ from the values at $\phi=45^{\circ}, 135^{\circ}$, corresponding to taking the change in the $y$ direction. The $r$ derivative is of course determined by subtracting the four values at $r_{i-1 / 2}$ from the four values at $r_{i+1 / 2}$. In obtaining the finite difference, one must also take into account the change in "Cartesian" direction of the $\theta$ and $\phi$ unit vectors across the pole, e.g., near $\theta=0^{\circ}$, a vector with positive $v_{\theta}$ at $\phi=45^{\circ}$ points in the direction opposite to that of a vector with the same $v_{\theta}$ at $\phi=225^{\circ}$. For the flows considered in the simulations reported here, the change in sign of the unit vectors is correctly accounted for by substituting $-v_{\theta},-v_{\phi},-B_{\theta},-B_{\phi}$ for $v_{\theta}, v_{\phi}, B_{\theta}, B_{\phi}$ at $\phi=135^{\circ}, 225^{\circ}$ in the difference equations. As shown in (18)-(24), viscous and resistive terms are included in the calculation at the pole (as in the calculation on the interior grid), primarily for numerical stability. In addition, smoothing of $\rho$ and $P / \rho^{\gamma}$ (by averaging nearby values) was performed at the poles to suppress shortwavelength fluctuations.

Once the pole values are calculated for $\phi=0$, pole values must also be calculated at the other values of $\phi$. These values must be obtained in order to perform calculations at all $\phi$ for the $\theta$ grid points nearest the poles. For the scalar variables $\left(\rho, P \rho^{-\gamma}\right)$ the values at the pole are independent of $\phi$ and thus given by their value at $\phi=0$. For the vector quantities, however, the values vary with $\phi$ because each different $\phi$ is related to a different Cartesian coordinate system. For example, when $\phi=0$, the vector $\left(0,0, B_{\phi}\right)$ with $B_{\phi}>0$ points in the $y$ direction; however, when $\phi=90^{\circ}$, a vector with these same components points in the negative $x$ direction. The spherical coordinate components of a vector $\vec{A}$ at a pole can be found for any $\phi$ from the components of $\vec{A}$ at $\phi=0$ by relating the spherical coordinate components of $\vec{A}$ to the Cartesian components. Thus

$$
\begin{aligned}
& A_{r}=A_{x} \sin \theta \cos \phi+A_{y} \sin \theta \sin \phi+A_{z} \cos \theta \\
& A_{\theta}=A_{x} \cos \theta \cos \phi+A_{y} \cos \theta \sin \phi-A_{z} \sin \theta \\
& A_{\phi}=-A_{x} \sin \phi+A_{y} \cos \phi
\end{aligned}
$$


At $\theta=(0, \pi)$, for any $\phi$,

$$
\begin{aligned}
& A_{\tau}= \pm A_{z} \\
& A_{\theta}= \pm A_{x} \cos \phi \pm A_{y} \sin \phi \\
& A_{\phi}=-A_{x} \sin \phi+A_{y} \cos \phi
\end{aligned}
$$

If $\left(A_{r 0}, A_{\theta 0}, A_{\phi 0}\right)$ are the components of $\vec{A}$ at a given pole for $\phi=0$,

$$
\begin{aligned}
& A_{x}= \pm A_{\theta 0} \\
& A_{y}=A_{\phi 0} \\
& A_{z}= \pm A_{r 0}
\end{aligned}
$$

Substituting (35)-(37) into (32)-(34), we obtain the components of the vector $\vec{A}$ for any $\phi$ in terms of $\left(A_{r 0}, A_{\theta 0}, A_{\phi 0}\right)$ in the form

$$
\begin{aligned}
& A_{r}=A_{r 0} \\
& A_{\theta}=A_{\theta 0} \cos \phi \pm A_{\phi 0} \sin \phi \\
& A_{\phi}=\mp A_{\theta 0} \sin \phi+A_{\phi 0} \cos \phi
\end{aligned}
$$

\subsection{Initial Conditions and Boundary Conditions}

The simulation starts with uniform flow in the positive $z$ direction, and uniform magnetic field in the negative $y$ direction (see Figure 1a). At time $t=0$, Io is inserted into the uniform flow. We then advance the simulation in time until the flow relaxes to a steady state. The simulations were performed (and all results are shown) in Io's rest frame.

There are two boundaries in the simulation: at Io's surface and at the outer boundary of the simulation region ( $r=10$ Io radii from the center of Io for most of the simulation runs). As stated in the introduction, we assume that Io has no intrinsic magnetic field. If this assumption is correct, in over 4 billion years the large Jovian magnetic field has certainly diffused into Io. On the other hand, the conductivity of Io and its ionosphere must be high enough to drive the field-aligned currents that create the perturbation fields observed by Voyager 1 [Acuna et al., 1981]. Because Io and its ionosphere must have a finite conductivity, we place no boundary condition on the magnetic field at Io, allowing the magnetic field to diffuse freely into Io. Io's conductivity is probably concentrated in its ionosphere, but for computational purposes the entire sphere is treated as a conductor. Thus the simulation can be thought of as being made up of two regions, one exterior and one interior to Io's surface. In the exterior region the full MHD equations are solved, while in the interior only the magnetic field is solved for using the resistive terms in equation (5). $\vec{B}$ is continuous across Io's surface, but $\rho, P \rho^{-\gamma}$, and $\vec{v}$ are subject to a boundary condition that represents the effects of Io's surface, atmosphere, and ionosphere. The requirement of a substantial ionospheric conductivity implies that Io's exobase must be above the surface over a significant portion of the satellite, so we assume that the torus plasma does not reach Io's surface. Accordingly we set $\vec{v}=0$ there and calculate $\rho$ and $P \rho^{-\gamma}$ assuming the surface and atmosphere behave like an impenetrable wall.

Allowing the field to diffuse all the way through the sphere presents us with the problem of dealing with the singularity in the equations at $r=0$. However, only the magnetic field is calculated inside the sphere, so we avoid this singularity by calculating $\vec{B}$ everywhere except at $r$ $=0$, and then calculating $\vec{B}$ at $\mathbf{r}=0$ using $\nabla \cdot \vec{B}=0$.

The resistivity, $\eta$, for the sphere is determined by requiring that the integrated conductivity of the sphere match the value for the integrated conductivity of Io's ionosphere. In the simulation the resistivity changes over 3 radial grid points near Io from the value which represents Io's resistivity to a smaller background value used throughout the simulation region in order to maintain numerical stability. The region of changing conductivity, plus the conductivity of the sphere itself, is used to mimic Io's ionosphere.

In contrast to the inner boundary at Io's surface, the outer boundary of the simulation is a computational boundary; it is required because of the finite size of the computer. Over some portion of the outer boundary it is necessary to specify the upstream conditions (representing the background flow), while for the rest of the outer boundary, conditions that are "free" (allowing inflow or outflow) are preferable. A somewhat arbitrary decision must be made on where the transition from upstream to free conditions occurs. Through experimentation we found that placing this transition near $\theta=135^{\circ}$ was generally best, i.e., resulted in the least effects on the interior flow (as shown in Figure $1 a, \theta=180^{\circ}$ is directly upstream of Io, and $\theta=0^{\circ}$ is directly downstream of Io). The free boundary condition was implemented by setting $\partial u / \partial r=0$ at the boundary, where $u$ represents any simulation variable. Increased resistivity was introduced for several grid points around the entire outer boundary to damp out any wave reflections. To ensure that our outer boundary did not produce unphysical effects, we ran several simulations with the outer boundary moved from $r=10$ to $r=15$ Io radii, and found no qualitative changes in the results described here.

We did not directly impose any symmetry on the solutions via the boundary conditions in the simulation. However, the initial conditions we used in the simulation imply that the scalar variables $\left(\rho, P / \rho^{\gamma}\right)$ must be symmetric and the vector quantities $(\vec{v}, \vec{B})$ must be either symmetric or antisymmetric, depending on the quantity and the symmetry plane considered. Verification that the solutions displayed the required symmetries served as a useful check on the calculation.

\subsection{Simulation Parameters}

Input parameters required for the simulation include the integrated conductivity (conductance) of Io's ionosphere, the Alfvén Mach number $\left(M_{A}\right)$, and the sonic Mach number $\left(M_{s}\right)$. The ratio of plasma pressure to magnetic pressure, $\beta$, is fixed by the choice of Mach numbers. $M_{A}$ for the plasma flow at Io is probably in the range 0.15 to 0.25 [Southwood et al., 1980]. $M_{S}$ in the Io torus is uncertain; $M_{S}=1.8$ for oxygen ions and $M_{S}=2.5$ for sulfur ions in the thermal plasma, assuming a background temperature of $100 \mathrm{eV}$ [Bagenal, 1985]. However, the flux of ions with energies $>6 \mathrm{keV}$ has not been measured, and their contribution to the pressure is probably significant ( $\mathrm{K}$. Khurana, personal communication, 1991). For example, assuming an aver- 
age mass number of 20 , if all of the ions with energies $>6 \mathrm{keV}$ gave the same contribution to the plasma pressure as a $1 \%$ population of $30 \mathrm{keV}$ ions, $M_{S}$ would be 1. One sees, then, that the torus plasma is sub-Alfvénic and probably either transonic or supersonic. Simulations at higher $M_{A}$ and lower $M_{S}$ (and thus higher $\beta$ ) presented fewer numerical difficulties and were less expensive to perform. In this paper we consider simulations with $M_{A}=0.33$ and 0.5 and $M_{S}=0.5$ and 1 . These choices mean that $\beta$ in our simulation is somewhat larger than is probable in the actual torus. While our values differ slightly from the Io torus parameter regime, they should be close enough to illuminate the important physical mechanisms involved.

The conductance of Io's ionosphere is unknown. Values ranging from 12 mhos to 126 mhos have been considered previously [Hill et al., 1983; Wolf-Gladrow et al., 1987]. We have performed simulations with ionospheric conductances ranging from 37 mhos to 130 mhos. Equation (1) shows that reduction of the flow speed near Io is determined by the product of Io's conductance and the Alfvén speed. Because we generally use Alfvén speeds in the simulation that are somewhat smaller than are likely to occur in the torus, the slowing of the flow near Io will be underestimated unless Io's conductance is correspondingly increased. This implies that the ionospheric conductance used in our simulation corresponds to a smaller ionospheric conductance for the actual torus parameter regime. Accordingly, for most of the simulation runs, we used relatively high values for Io's conductance (130 mhos).

The simulation code used either a $21 \times 31 \times 38$ or a $31 \times 31 \times 38$ grid $(r, \theta, \phi$ grid dimensions). This corresponds to $\Delta r=1 / 2 R_{\mathrm{Io}}, \Delta \theta=6^{\circ}$, and $\Delta \phi=10^{\circ}$, with a radial extent of the simulation of either 10 or $15 R_{\mathrm{Io}}$. The time step limit for methods using explicit temporal differencing for advective problems is the well-known Courant-Friedrichs-Levy (CFL) condition [e.g. Richtmyer and Morton, 1967], $\Delta t \leq \Delta l /|v|$, where the cell size is $\Delta l$ and $v$ is the fastest physical speed in the system. For diffusion the time step limit is $\Delta t \leq \Delta l^{2} /(2 D)$, where $D$ is the diffusion coefficient. In spherical coordinates these relations hold with cell sizes for the $r, \theta$, and $\phi$ directions of $\Delta r, r \Delta \theta$, and $r \sin \theta \Delta \phi$. The most severe time step limitation arises in the $\phi$ direction near the poles. The limitation can be especially severe for diffusion because the time step limitation depends on the square of the cell size. For $\Delta \theta \approx \Delta \phi$, the time step limitation is $\Delta t \leq r^{2} \Delta \phi^{4} /(2 D)$, so halving the angular grid size requires 8 times as many time steps if diffusion is the dominant process limiting the time step. In most of the regions of our simulation the diffusion terms in the equations are unimportant with regard to the time step because the diffusion coefficient is small. However, within Io and nearby, the time step limitation for diffusion of magnetic field into Io (where the resistivity $\eta$ acts as the diffusion coefficient) can become important because the conductivity of Io can be relatively small (relatively large $\eta$ ). Close to and inside Io, the cell size in the $\phi$ direction also becomes small. Thus the time scale for diffusion of field inside Io can control the time step of the entire calculation. In our initial simulation runs we found that the magnetic field changed very lit- tle inside the sphere. This is not unexpected, as WolfGladrow et al. [1987] also found that the field inside Io was essentially the background field for a wide range of conductivities. For most of the simulation runs we did not advance the magnetic field for the grid points inside the sphere. We found no qualitative difference in the results obtained with this approximation, which achieved an enormous savings in the computation time.

\section{Results}

After the conducting Io is placed in the uniform plasma flow and magnetic field, a quasi-steady state configuration forms after 2-3 Alfvén travel times (the travel time is the time for a wave to propagate from one boundary to the other). Individual simulation runs were performed for as much as 6 times longer with no qualitative changes in the final results.

Display of data from three-dimensional simulations presents a number of difficulties. Generally one must resort to looking at different planes in the simulation for at least some of the quantities of interest. In many of the figures we show data from the $y z$ plane or the $x z$ plane. The $y z$ plane cuts through Io and is parallel to the initial flow and magnetic field, whereas the $x z$ plane cuts through Io and is parallel to the initial flow but perpendicular to the initial magnetic field (see Figure $1 a)$. The $x z$ plane can be thought of as Io's equatorial plane. We also show tracings of the magnetic field and flow velocity, and vector plots of the flow velocity and parallel current density, in three dimensions.

\subsection{The Alfvén Wing}

As described in the introduction, the formation of the Alfvén wing is a fundamental property expected from both analytic theory and observations for the interaction of the torus plasma with Io, and a feature we expect to see in the simulation. We first examine the magnetic field. Figure 2 shows three-dimensional tracings of the magnetic field in the vicinity of Io for a simulation with $M_{A}=0.5$. Io is the sphere drawn with dashed lines. We see that the field hangs up around Io. Draping of the magnetic field is a basic feature that one expects for the interaction of a flowing, magnetized plasma with a conducting obstacle. We can get a rough idea of where the Alfven wing lies by examining the inclination of the magnetic field. Figure 3a shows tracings of the magnetic field in the $y z$ plane for the same simulation as in Figure 2. We see that the magnetic field in this plane bends away from the vertical on either side of Io in the vicinity of the Alfven characteristics, indicated by the heavy parallel lines drawn attached to Io. In this and subsequent diagrams the Alfvén characteristics are defined by the unperturbed Alfvén Mach number. Note that the field bends downstream of Io, as well as where the field lines thread Io. As the Alfvén characteristics occur at an angle $\tan ^{-1} M_{A}$ to the background field, we expect that if we run a simulation with lower $M_{A}$, the inclination of the magnetic field should be less. Figure $3 b$ shows tracings of the field for the same plane as in Figure $3 a$ but for a simulation with $M_{A}=0.33$. The magnetic field is less inclined in Figure $3 b$ than in Figure 


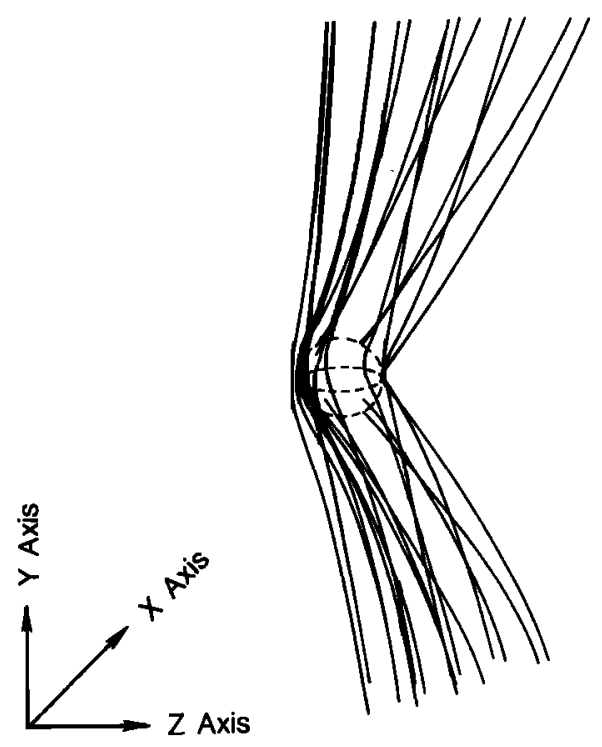

Fig. 2. Magnetic field lines near Io for a simulation with $M_{A}=0.5, M_{S}=0.55$. Io is the sphere drawn with dashed lines. The magnetic field drapes around Io; field lines intersecting Io are bent the most. The field lines were calculated by integrating the magnetic field values in the simulation in three dimensions.

$3 a$, again near the Alfvén characteristics. Both Figure $3 a$ and Figure $3 b$ show a piling up of field lines slightly downstream of the Alfvén characteristics attached to Io. As described later in this paper, increases in the magnetic field strength caused by both slow and fast mode perturbations occur in this plane (see section 3.3).

Tracings of the total magnetic field are informative for examining effects on the field, but they do not necessarily give an accurate representation of where the Alfvén wing lies because the Alfvén characteristics indicate where the perturbations to the field are, and do not indicate the direction of the total magnetic field. Thus a more appropriate way to look for the Alfvén wing is to identify the regions of standing Alfvén wave perturbations, i.e., the regions where field-aligned currents flow [e.g., Southwood et al., 1980; Neubauer, 1980]. Figure 4 shows field-aligned current density vectors above a small threshold (about $10 \%$ of the largest vectors) in the same simulation as Figure 2, plotted in three dimensions. Figure $4 a$ shows the vectors viewed upstream from Io; the plasma flow is into the plot, and the initial magnetic field (not shown) is in the negative y direction, pointing downward in the plot. The accompanying diagram is taken from Neubauer [1980] and shows the expected sense of the parallel currents generated at Io. The currents flow toward Io on the left-hand side and away from Io on the right-hand side. (Note that there are also perpendicular currents connected with the Alfvén wing [Wright and Southwood, 1987].) The sense of the currents in the simulation agrees with the result from analytic theory. Figure $4 a$ also shows that the $y z$ plane (the plane for which the magnetic field tracings are shown in Figure 3) is the plane across which the field-aligned currents reverse direction; the field-aligned currents are exactly zero in that plane. The currents are exactly antisymmetric, as would be expected for the initial condition we have used (Figure 1a); however, the graphics program introduced some slight asymmetries. The view is slightly tilted, so the vectors above Io appear slightly longer than those below. To make the plot less dense, vectors were plotted only at positions with $z \leq 0$; however, the plotting program did not plot all the vectors at $z=0$, resulting in a few more vectors being plotted below Io than above Io. Asymmetry between the right and left halves of Figure $4 a$ is just the result of the vectors being plotted with the tail beginning at the grid point, so the heads of antisymmetric vectors appear in different vertical locations.

Figure $4 b$ shows field-aligned current density vectors

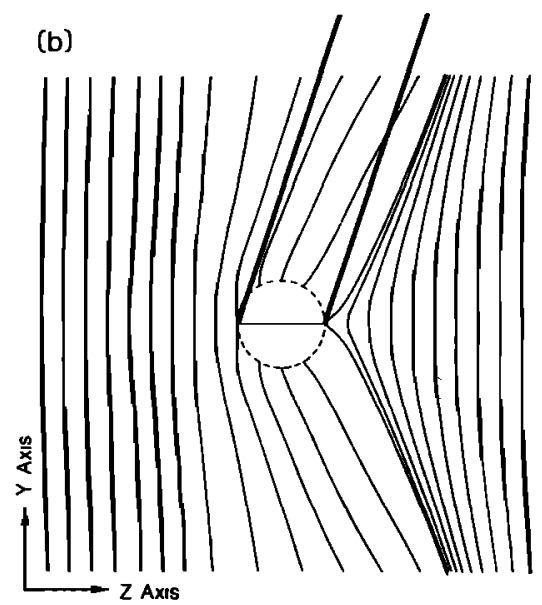

Fig. 3. (a) Tracings of the magnetic field in the $y z$ plane for the same simulation as Figure 2 . The direction of the Alfvén characteristics (based on the initial simulation parameters) in the vicinity of Io is indicated by the heavy parallel lines. (b) The same as Figure $3 a$ but for a simulation with $M_{A}=0.33$. The bend in the total magnetic field is less than in Figure $3 a$. As the Alfven characteristics occur at an angle $\tan ^{-1} M_{A}$ to the background field, the characteristics bend less relative to the background field for cases of lower $M_{A}$. Formally, the Alfvén characteristics describe where the perturbations to the magnetic field should occur. Figure 3 shows that they also indicate where the bend in the total magnetic field occurs. 


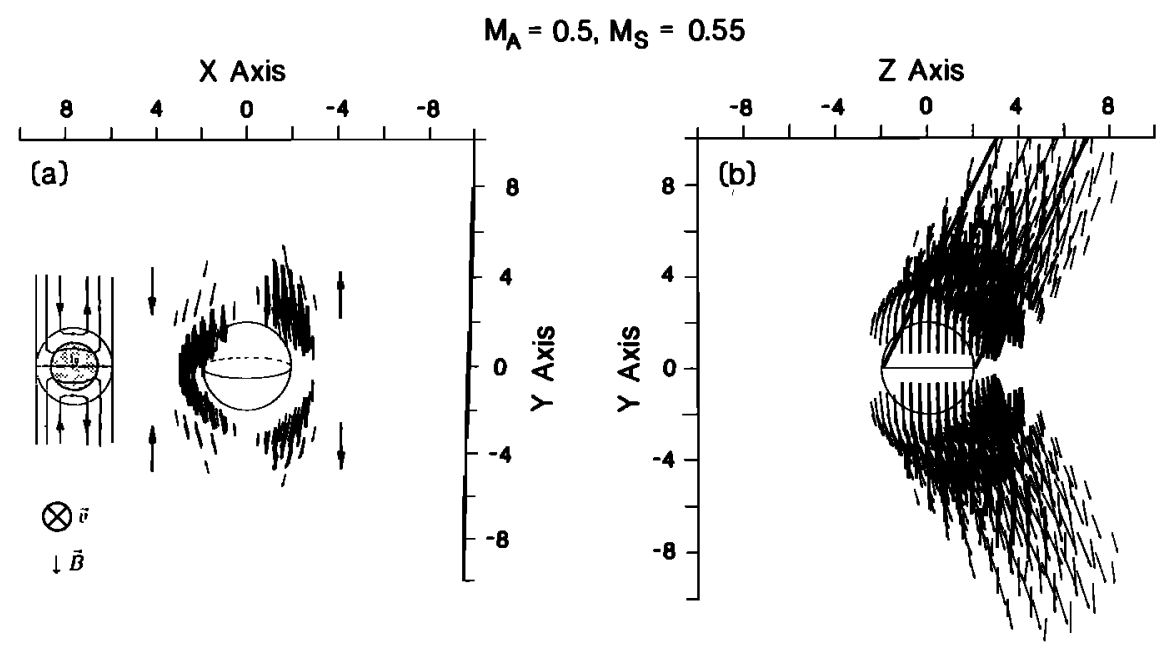

Fig. 4. (a) On the left, from Neubauer [1980], current streamlines near Io, and on the right, current density vectors parallel to the magnetic field, within $5 R_{\text {Io }}$ of Io, for the same simulation as Figure 2. Vectors above a small threshold are plotted in three dimensions and are viewed from upstream of Io; only the vectors occurring upstream of Io are plotted. For this and all succeeding vector plots, the magnitude of the vector field at a given point is indicated by the length of the vector. The parallel current density vectors in the simulation agree with the expected streamlines. The currents are exactly asymmetric both above and below the figure and between the right and the left halves, but slight asymmetries appear in the plot as a result of the graphics display (see text). (b) Field-aligned current density vectors viewed from the $-x$ direction. Current density vectors for $x \leq 0$ are plotted, eliminating one "wing". Solid lines represent Alfvén characteristics attached to Io. The region of field-aligned current generation corresponds to the expected Alfvén wing region.

viewed from the negative $x$ direction (looking inward toward Jupiter). Only the vectors between the viewer and the center of Io have been plotted; as can be seen in Figure $4 a$, the current flows in the opposite direction on the other side of Io. We have again drawn Alfvén characteristics (based on initial conditions) attached to Io in the $y z$ plane. The simulation allows the flow to react nonlinearly to the presence of Io, so the Alfvén speed itself changes in the near vicinity of Io. Despite the nonlinear effects, Figure $4 b$ shows that the field-aligned current generation occurs along the characteristics defined by the background values. However, the region of current generation is somewhat larger than Io, extending both slightly upstream and downstream of Io.

The purpose of Figure $4 a$ is to show the direction of the parallel currents close to Io; the decrease in the size and number of vectors for $y> \pm 4\left( \pm 2 R_{\mathrm{Io}}\right)$ should not be interpreted as a decrease in the strength of the parallel current density. Only vectors upstream of lo were plotted in Figure $4 a$; most of the Alfvén wing lies downstream of Io $(z>0)$ and was not plotted. Vectors with $z<0$ and at larger distances from Io in the $\pm y$ direction are also farther from the center of the Alfvén wing, as can be seen in Figure $4 b$. Figure $4 b$ actually shows that in the near vicinity of Io, the field-aligned current density vectors at a given distance from the center of the Alfvén wing are of roughly constant strength, regardless of their distance from lo. However, on larger scales than shown in the figure $\left(r>6-7 R_{\mathrm{Io}}\right)$, the fieldaligned currents do decrease in magnitude. This is a numerical effect, caused by the increased spacing of the grid with distance. At large radial distances from Io the Alfven wing is resolved over fewer grid points, so the field-aligned currents partially close in perpendicu- lar currents that are present because of truncation errors in the algorithm.

\subsection{Flow in the Vicinity of Io and the Alfvern Wing}

Figure $5 a$ shows plasma flow in the $y z$ plane (same plane as Figure 3) for a simulation with $M_{A}=0.5$. Alfvén characteristics attached to Io are again drawn as reference. We see that within the Alfven wing the flow is slower than the upstream flow, and the flow reaccelerates downstream of Io. The flow slows because the field lines in the Alfvén wing are part of the flux tube connected to Io (see Figure $3 a$ ), and because the ionospheric conductivity of Io is high, the field lines diffuse quite slowly through Io. The plasma on the flux tubes linked to Io then must also move very slowly. Figure $5 b$ is the same as Figure $5 a$ but for a simulation with $M_{A}=0.33$; once again the region of reduced flow is associated with the Alfvén characteristics. Figure $5 b$ also shows a vortical structure near the reduced flow region in the Alfvén wing. This structure does not appear to be caused directly by the Alfvén mode, but may appear as a result of the strongly reduced flow velocity in the Alfven wing. The appearance of this structure is somewhat dependent on both the local magnetic and fluid Reynolds numbers; thus it may be a numerical effect.

One also expects that the Alfvén wings will form obstacles to the flow even well above and below Io as suggested by Neubauer [1980] and Barnett [1986]. A simple model of the Alfven wing serves to illustrate this point. Consider the Alfvén wing to be a cylinder of radius $1 R_{\mathrm{Io}}$, connected to Io and tilted at $\tan ^{-1} M_{A}$. The intersection of a given plane above Io with this cylinder is an ellipse; we refer to this as the "Alfvén ellipse". Figure 


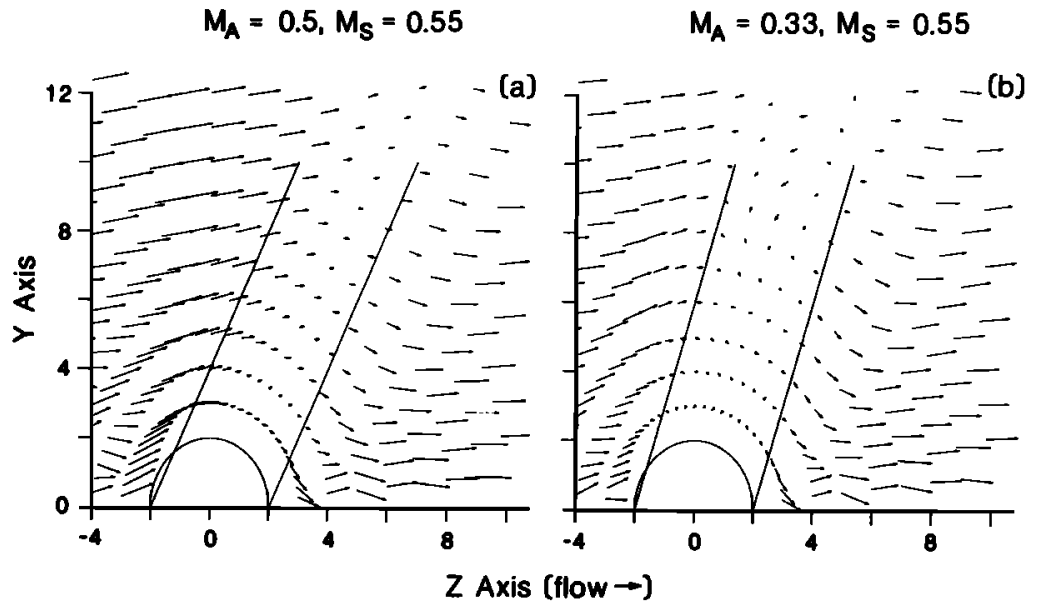

Fig. 5. (a) Plasma flow in the $y z$ plane for the same simulation as Figure $2 ; \Sigma_{\text {Io }}=130$ mhos. The solid lines represent Alfvén characteristics attached to Io. Reduction of the plasma flow occurs within the Alfvén wing. (b) The same as Figure 5a, but for $M_{A}=0.33\left(\Sigma_{\mathrm{Io}}=130\right.$ mhos). Lowering $M_{A}$ causes the region of reduced flow to move, showing that the flow reduction is indeed associated with the Alfvén wing.

$6 a$ shows three-dimensional flow vectors from a simulation with $M_{A}=0.5$, in a "slab" above Io, $1 / 2 R_{\mathrm{Io}}$ thick (bounded by the planes at $y=2 R_{\mathrm{Io}}$ and $y=2.5 R_{\mathrm{Io}}$ ). Because of the coordinate system used in the simulation, we cannot show an arbitrary "plane" above Io as the grid points do not all lie in a plane; therefore we must show a slab of finite thickness. The dotted circle shown is a sphere drawn at Io's position, and the solid lines show the Alfvén ellipse at $2.25 R_{\mathrm{Io}}$ above Io (the midpoint of the slab of flow vectors). The flow is viewed from above Io (the sphere appears smaller than the Alfvén ellipse because it is farther away from the viewpoint). Figure $6 a$ shows that the smallest flow vectors occur within the Alfvén ellipse and that the flow diverts near the Alfvén ellipse and closes behind it. Figure $6 b$ is the same as Figure $6 a$ except that $M_{A}=0.33$ in the simulation, and, to differentiate the projected position of the Alfvén ellipse and Io for this lower $M_{A}$ case, a slab of flow vectors at a location slightly farther above Io (bounded by the planes at $y=2.5 R_{\mathrm{Io}}$ and $y=3.0 R_{\mathrm{Io}}$, with the Alfvén ellipse at $2.75 R_{\mathrm{Io}}$ ) is shown. Figure $6 b$ again shows that the flow diversion is around the Alfvén wing and the flow is most reduced within this region.

The model for the Alfvén wing that we have described has some limitations for interpreting the simulation data. The flow vectors plotted in Figure 6 are not coplanar with the Alfven ellipse, but are in a range of $\pm 1 / 4 R_{\mathrm{Io}}$ around it, so the flow vectors plotted near

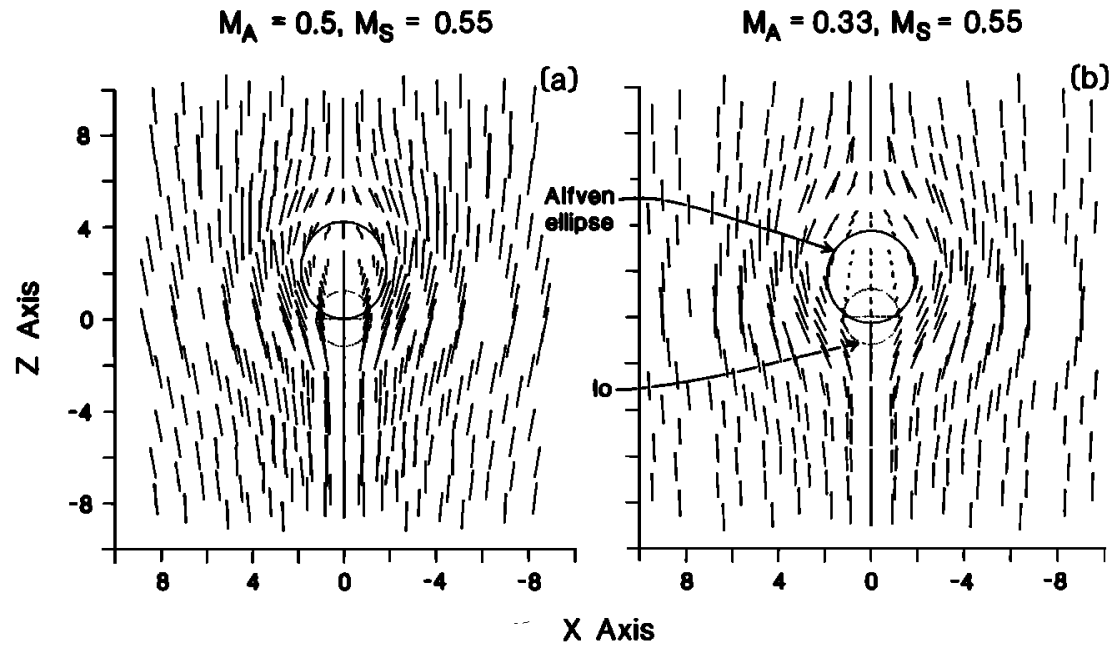

Fig. 6. (a) Plasma velocity vectors in three dimensions, viewed from above Io for the same simulation as Figure 2. $\Sigma_{I_{0}}=130$ mhos. The vectors are plotted in a "slab" between 2 and $2.5 R_{\text {Io. The flow in }}$ relation to the Alfvén ellipse at $R=2.25$ (solid line; see text) and Io (dotted lines) is shown. The sphere representing Io appears smaller than the Alfvén ellipse because it is farther away from the viewpoint. The smallest flow vectors occur within the Alfvén ellipse, and the flow diverts near the ellipse and closes behind it. (b) The same as Figure $6 a$ except that $M_{A}=0.33$, and vectors between 2.5 and $3 R_{\mathrm{Io}}$ are shown in relation to the Alfvén ellipse at $2.75 R_{\mathrm{Io}}$. The flow reduction and diversion is clearly associated with the Alfvén wing. 
the ellipse may actually be slightly upstream or downstream of the Alfvén wing at their actual height above Io. Furthermore, the obstacle to the flow is expected to be larger than the schematic ellipse because, as is evident from Figure $4 b$, the "diameter" of the Alfven wing is actually larger than an Io diameter. Despite these limitations, Figure 6 shows that the Alfvén ellipse provides a useful approximation as the obstacle to the flow at a given distance above Io. Figures 5 and 6 show that the Alfvén wing is not an impenetrable obstacle; within its boundaries the flow is slowed but not stopped.

Figures 5 and 6 also show that the flow retardation is greater for lower $M_{A}$ (both runs used the same value for Io's ionospheric conductance, $\Sigma_{I_{0}} \approx 130$ mhos). This result is consistent with equation (1), i.e., more flow reduction for higher Alfvén speed; however, to test (1) we should look at the flow electric field $(E=\vec{v} \times \vec{B})$ rather than the velocity. Examining the magnitude of $E$ in the Alfven wing in the simulations, we find that $\alpha=E_{\mathrm{Io}} / E_{u}$ is $\alpha_{c 1} \approx 0.09$ in the $M_{A}=0.33$ simulation and $\alpha_{c 2} \approx 0.17$ in the $M_{A}=0.5$ case. The reduction factor expected from equation (1) for $M_{A}=0.33$ ( $\Sigma_{A} \approx 4.4$ mhos) is $\alpha_{e 1} \approx 0.06$ and, for $M_{A}=0.5$ $\left(\Sigma_{A} \approx 6.6\right.$ mhos), $\alpha_{e 2} \approx 0.09$. The ratio $\alpha_{c 2} / \alpha_{c 1}$ is close to the expected ratio $\alpha_{e 2} / \alpha_{e 1}$ (1.8 instead of 1.5), but reduction of $E_{u}$ is less in both simulations than expected (by about a factor of 1.5-2). Although this shows reasonably good agreement with equation (1), we note that Io is resolved over only 4 radial cells in the simulation. Some or all of the deviation may be caused by the presence of additional numerical dissipation in the differencing algorithm that makes the effective $\Sigma_{\text {Io }}$ less than the nominal value.

From equation (1) we would also expect that the flow velocity within the Alfvén wing should increase (i.e., be less perturbed) if the conductance of Io's ionosphere is reduced. Figure 7 shows the flow in the $y z$ plane for a simulation with $M_{A}=0.5$. The parameters for this

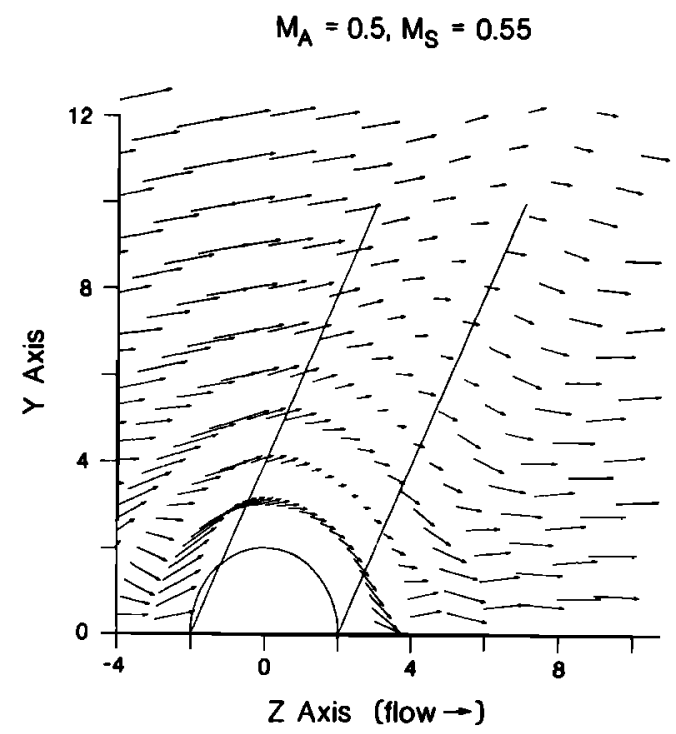

Fig. 7. The same as Figure 5a, except that Io's ionospheric conductance for this simulation was lower ( 37 mhos). Flow reduction in the Alfvén wing is not as great as for the higherconductivity case.

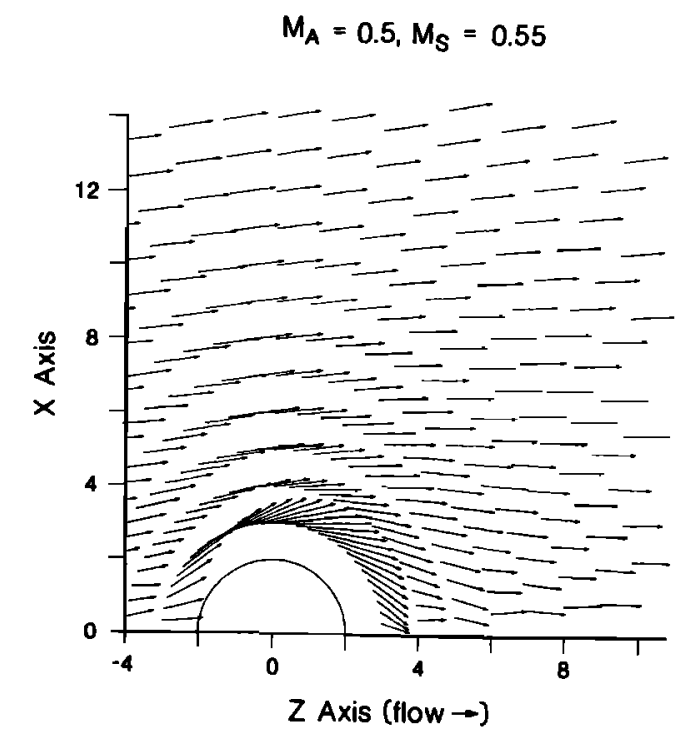

Fig. 8. The plasma velocity in the $x z$ (equatorial) plane for the same simulation as Figure $5 a\left(\Sigma_{\mathrm{Io}}=130 \mathrm{mhos}\right)$. The flow diverts smoothly around Io, is faster on the flanks, and is reduced just downstream behind Io. The flow is reaccelerated farther downstream of Io.

simulation are the same as for Figure 5a, except that the ionospheric conductance was reduced from 130 to 37 mhos. The flow in the Alfven wings is faster in Figure 7 than in Figure 5a, as expected. The expected $E_{u}$ reduction factor for this simulation, $\alpha_{\mathrm{e} 3} \approx 0.26$, is again smaller than the computed value $\left(\alpha_{c 3} \approx 0.39\right)$ but the ratio $\alpha_{e 3} / \alpha_{e 2} \approx 2.9$ is close to the computed value $\left(\alpha_{c 3} / \alpha_{c 2} \approx 2.3\right)$. Again reasonably good agreement with equation (1) is found, and the deviation is consistent with an effective $\Sigma_{\text {Io }}$ that is less than the nominal value because of numerical dissipation.

For completeness, we also show the flow in the $x z$ (Io's equatorial) plane (Figure 8) for the simulation of Figure $5 a\left(\Sigma_{\mathrm{Io}}=130 \mathrm{mhos}\right)$. We see that the flow diverts around Io smoothly and is accelerated on the flanks, similar to what was seen in Figure 6 for flow around the Alfvén wing. The flow slows immediately downstream of Io but is reaccelerated further downstream.

Thus far many of the effects on the flow near Io that we have described are caused by the bending of the magnetic field near Io. The plasma interaction with Io not only bends the magnetic field (as expected from Alfvénic disturbances) but also changes the field strength. Figure $9 a$ shows the magnetic pressure $\left(B^{2} / 2\right)$ in the $x z$ plane, represented as a surface for the simulation with $M_{A}=0.5, M_{S}=0.55$. For this and all succeeding surface plots shown, Io is located at the center of the diagram and has a radius of 2 in the units shown. There is a large increase in the field strength in front of Io where the magnetic field hangs up. The field strength decreases downstream of Io, because the field diffuses through Io slowly. Figure $9 b$ is the same as Figure $9 a$ except that the plasma pressure is depicted. The pressure upstream of Io changes little, but the plasma pressure increases slightly downstream of Io. Changes to the magnetic and plasma pressures indicate that the 
$M_{A}=0.5, M_{S}=0.55$

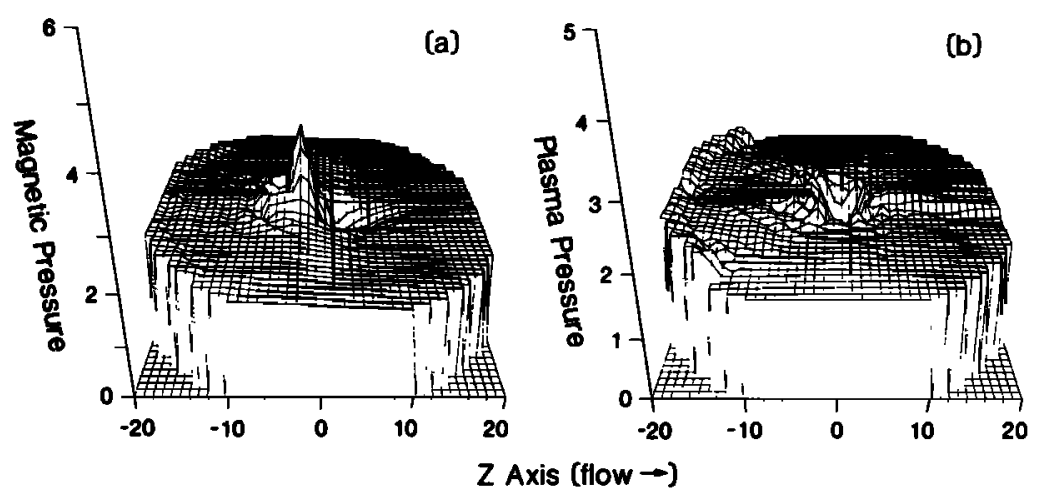

Fig. 9. (a) A surface representation of the magnetic pressure in the $x z$ plane for the same simulation as Figure 8. The height of the surface at each point in the plot represents the magnitude of the magnetic pressure at that point. For all of the surface plots shown in this paper, the polar coordinate data in the plane are interpolated onto a Cartesian grid before the surface is plotted; Io is in the center of the plot and has a radius of 2 in the units shown. The large increase in magnetic pressure in front of Io is caused by the magnetic field hanging up as the field slowly diffuses into Io. The magnetic pressure decreases downstream of Io. (b) The same as Figure $9 a$ but for the plasma pressure. The small ripples arise from numerical fluctuations. The slight increase in plasma pressure in Io's wake (and the decreased magnetic pressure seen in Figure 9a) is associated with the pressure-enhancing slow mode wave (see text and Figure 10).

disturbances caused by Io are not purely Alfvénic. We describe these effects in the next section.

\subsection{Slow and Fast Mode Perturbations}

As mentioned in the introduction, most previous work on the torus plasma-Io interaction has concentrated on the Alfvén mode. Our simulations indicate that the other MHD modes must also play a role. Linker et al.
[1988] showed that standing slow mode perturbations develop in the flow; Figure 10, adapted from that paper, shows the plasma pressure in the $y z$ plane for a simulation with $M_{A}=0.5$ and $M_{S}=1.0$. Figure $10 a$ shows a surface representation, and Figure $10 b$ shows contours. Io is in the center of both plots and has a radius of 2 in the units shown. A region of decreased plasma pressure, approximately connected to Io, devel-

$$
M_{A}=0.5, M_{S}=1.0
$$
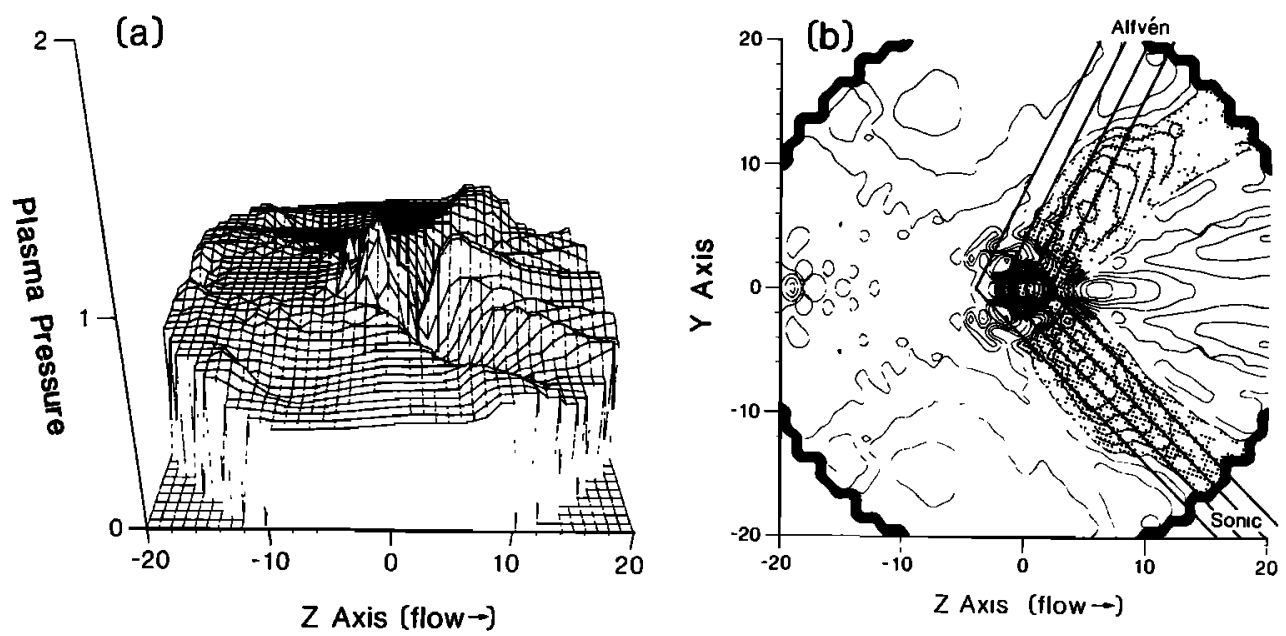

Fig. 10. (a) The same as Figure $9 b$, except that the plasma pressure in the $y z$ plane is depicted and $M_{S}=1$ for this simulation [after Linker et al., 1988]. A rarefaction in pressure, associated with a pressure-decreasing slow mode wave attached to Io, creates the "trough" in pressure shown. An increase in magnetic pressure is associated with this trough. (b) The same as Figure 10a except that the pressure is represented with contour lines. Io is in the center of the plot. The region of decreased plasma pressure has been shaded. Alfvén lines are drawn in the top half of the figure; sonic lines (corresponding to a slow mode perturbation propagating nearly parallel to $\vec{B}$ ) are drawn in the bottom half of the figure. The trough in plasma pressure attached to Io, as well as the region of increased plasma pressure behind Io, aligns approximately with the sonic lines. 
ops downstream (regions of decreased plasma pressure are shaded in Figure 10b). Further downstream, the plasma pressure is enhanced within a triangular region behind Io. The magnetic pressure has perturbations opposite in sign to the plasma pressure perturbations (the increase in the magnetic pressure associated with the slow mode plasma pressure rarefaction is evident in the magnetic field tracings depicted in Figures $3 a$ and $3 b$; also see Figure 3 of Linker et al. [1988]). The Alfvén mode only bends the magnetic field and does not perturb the magnetic or plasma pressures; thus the perturbations shown in Figure 10 must be related to one of the other MHD modes. The MHD mode that produces perturbations of opposite sign in the magnetic and plasma pressures is the slow mode. Unlike the Alfvén mode, the slow mode group velocity is not limited to propagation strictly parallel to the magnetic field; however, the slow mode is guided along the field. Along the magnetic field the slow mode group velocity is the sound speed (when $\beta<6 / 5$ ), so one would expect a standing slow mode perturbation to have characteristics close to sonic lines (tilted at $\tan ^{-1} M_{S}$ to the background field). Figure $10 b$ shows the relation of the rarefaction region to Alfvén characteristics (top set of parallel lines) and sonic characteristics (bottom set of parallel lines). Both the rarefaction region attached to Io and the pressure enhancement behind Io are closely associated with the sonic lines, as would be expected for a slow mode perturbation. We have found this to be true for all the simulation runs we have performed, i.e., the location of the rarefaction and enhancement of pressure follows the sonic lines radiating from Io for different choices of $M_{S}$. Figure 9 shows that the "width" of the slow mode perturbations corresponds to approximately an Io diameter.

Recently, Wright and Schwartz [1990] have argued for the existence of the standing slow mode perturbation using analytic theory. Examining the conservation of mass, momentum, and energy, they show that a purely Alfvénic disturbance will not satisfy the continuity relations. They point out that slow mode waves that either increase or decrease the plasma pressure can exist but that rarefaction waves can help to restore the missing fluxes in the continuity relations. Here we note that in our simulation, both rarefactions and compressions with slow mode phase relations form. However, the rarefaction in our simulation (which Wright and Schwartz compared their results to) is the much stronger perturbation ( $50 \%$ change in pressure for the rarefaction and $10 \%$ change for the enhancement).

The slow mode has the property that the total (magnetic plus plasma) pressure is constant. Figure 11a shows the total plasma pressure for the same simulation as Figure 10; we see that (in addition to a large increase in front of Io) there is a slight increase in the total pressure in the vicinity of the Alfven wings. The association of the increase in total pressure with the Alfven lines and not the sonic lines is evident in Figure $11 b$ which gives total pressure contours for the data of Figure 11a. We have found this to be true for the other runs we have performed; for example, Figure 11c shows contours of the total pressure for the run with $M_{A}=0.33$. Increases in the total pressure are indicative of a fast mode wave; therefore fast mode waves, propagating nearly parallel to $\vec{B}$, are responsible for these perturbations (there must be some oblique propagation for compression to occur).

We believe that the fast mode signature arises because as one cuts across the Alfvén wing in the $x$ direction, the field-aligned currents must reverse direction across the $y z$ plane (see Figure $4 a$ ). The symmetry of the plasma flow and magnetic field in this plane is such that $\vec{v}$ and $\vec{B}$ have only $y$ and $z$ components; there are no components out of the $y z$ plane (in a sense the simulation is quasi-two-dimensional there). Linear theory for the shear Alfvén (or intermediate) wave [e.g., Siscoe, 1983] shows that if the wave vector $\vec{k}$ and the background field
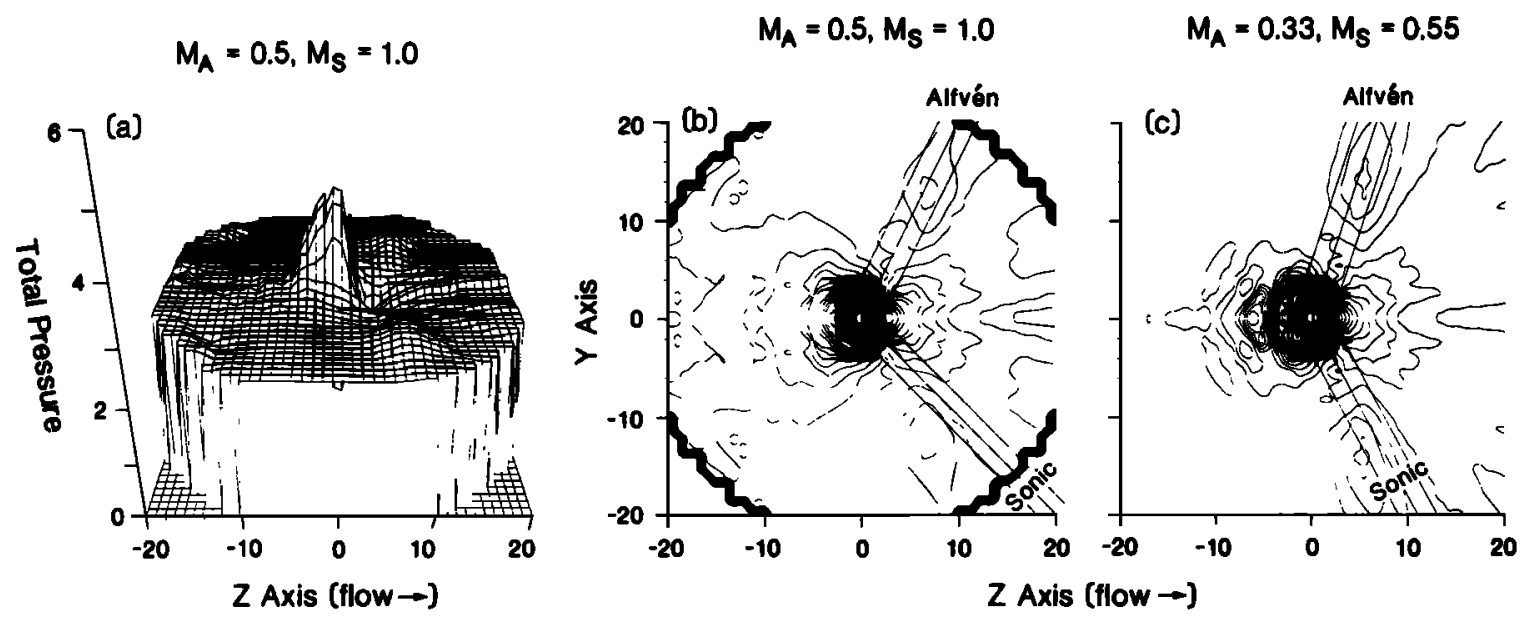

Fig. 11. (a) Total (plasma plus magnetic) pressure in the $y z$ plane for the same simulation as Figure 10, depicted as a surface. The total pressure increase in front of lo is driven primarily by the increase in magnetic pressure there (e.g., Figure 9a). A much smaller increase in total pressure can be seen slightly downstream of (above and below) Io's position. An increase in total pressure is indicative of a fast mode wave. (b) The same data as Figure 11a, depicted with contours. The increase in total pressure is clearly associated with the Alfvén lines, rather than the sonic lines, and is consistent with fast mode waves propagating nearly parallel to $\vec{B}$. (c) The same as Figure 11b, except for a simulation with $M_{A}=0.33, M_{S}=0.55$. Changing the Alfven Mach number causes the spatial location of the total pressure perturbation to change. Again, the perturbation is associated with the Alfvén lines. 
define one plane, then the velocity and magnetic field perturbations for the shear Alfven wave are out of this plane. Therefore the only Alfvén wave that can exist in the $y z$ plane is a "degenerate" fast mode wave; i.e., the fast mode propagating parallel to $\vec{B}$ and the Alfven wave with $\vec{k}$ along $\vec{B}$ are the same wave, and no fieldaligned currents can exist in the $y z$ plane (this can also be seen by examining $\nabla \times \vec{B}$ in the $y z$ plane and noting that $B_{x}, \partial B_{y} / \partial x$, and $\partial B_{z} / \partial x=0$ there). However, just because the shear Alfven wave does not exist in the $y z$ plane doesn't mean that the field will not bend there. On the contrary, the magnetic field lines in this plane cannot be diverted around Io, so they must bend (as shown in Figure 3), and therefore fast mode waves must be launched.

Because the amplitude of the fast mode perturbation peaks away from Io, we considered the possibility that this feature was caused by wave reflection from the outer boundary. Figure $11 c$ is from a simulation that extended to $15 R_{\mathrm{Io}}$ ( $r=30$ in the units of the figure). The data were plotted only out to $10 R_{\mathrm{I}}$ for easier comparison with Figures $11 a$ and $11 b$. The perturbation is located in approximately the same place in the larger simulation, so the fast mode signature does not appear to be caused by the outer boundary.

The fast mode perturbation shown in Figure 11 is much weaker in amplitude than the slow mode; this may be related to its more rapid propagation speed that can spread disturbances out more efficiently. Although the coordinate system of the calculation makes determining the "width" of the fast mode signature difficult, it appears to be more closely (but not completely) confined to the $y z$ plane than the slow mode perturbations (which have approximately the same dimension as Io). In fact, because the largest amplitude of the disturbance occurs far away from both Io and the equatorial plane, the resolution of the simulation results presented here does not allow us to rule out the possibility that if much higher resolution results were available, we would find that the fast mode was almost exclusively confined to the $y z$ plane alone, and that for the real interaction the fast mode signature might only occur over an infinitesimal region of space. However, we do not believe that this is the case. The fast mode signature is likely to arise only near those flux tubes whose field lines diffuse directly into Io's ionosphere (as opposed to those that are advected around it), but these flux tubes should constitute a finite region of physical space (albeit one that is smaller than Io's diameter).

While we have generally used linear arguments to explain the simulation results, the perturbations occurring in the simulation are clearly nonlinear. Figure $12 a$ shows the Alfvén speed $\left(V_{A}\right)$ in the $y z$ plane for the $M_{A}=0.5, M_{S}=1.0$ simulation; Figure $12 b$ shows the sound speed $\left(C_{S}\right)$ for the same case. Because the density decreases and the magnetic field increases in the rarefaction region, the Alfven speed increases. The pressure decreases in the rarefaction region faster than the density, so the sound speed decreases there. In the pressure enhancement region behind Io, the sound speed increases slightly, and the Alfvén speed remains relatively flat.

The different wave modes tended to occur in the same spatial regions of the simulation. For the $M_{A}=0.5$, $M_{S}=0.55$ case, where the sonic and Alfvén Mach numbers are very close together, this is unavoidable. However, even when the sonic and Alfvén Mach numbers were fairly far apart $\left(M_{A}=0.5, M_{S}=1\right)$, the spatial regions affected by different wave modes clearly overlapped near Io, although enough spatial separation occurred farther away from Io that regions affected by the individual modes could be distinguished. The overlap occurs naturally for perturbations generated by an obstacle with finite size (Io). The reason the total pressure (fast mode) perturbation shown in Figure 11 peaks away from Io may be related to the spatial overlap near Io of the much stronger slow mode perturbation with the fast mode. Farther away from Io, where the fast and slow modes have begun to separate, the total pressure perturbation is stronger.

Most analytic solutions have concentrated on the Alfvén mode. The justification for this approach is that the different MHD modes have different wave speeds, so the

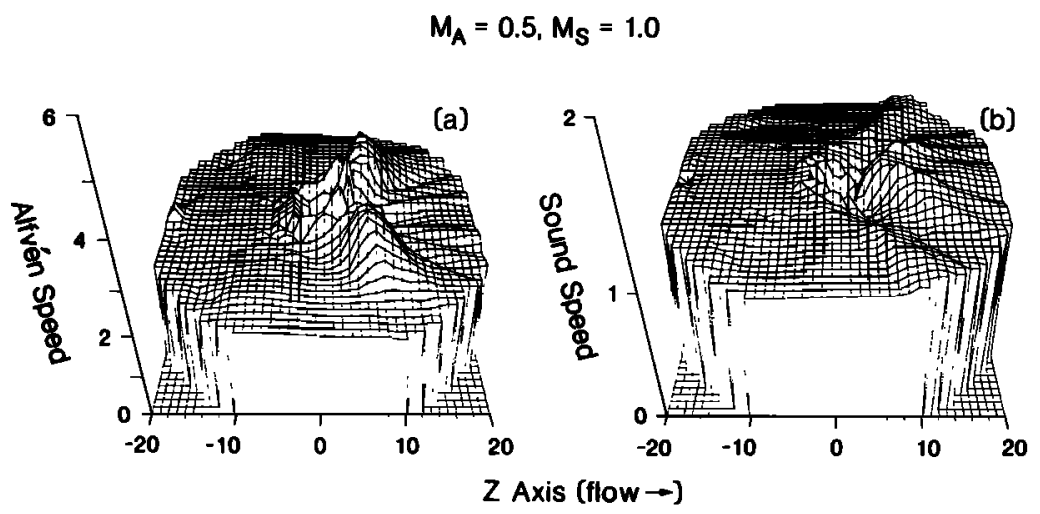

Fig. 12. (a) The Alfvén speed in the $y z$ plane for the same simulation as Figure 10. The perturbations in the simulation greatly affect the Alfvén speed. The increase in the Alfvén speed downstream of (above and below) Io is predominantly caused by the slow mode rarefaction wave (Figure 10), which increases the magnetic field and decreases the density in this region. (b) The same as Figure 12a except the sound speed is depicted. The sound speed is also affected by the MHD perturbations generated. The sound speed decreases at the location of the slow mode rarefaction. The slight increase in the sound speed just upstream of the decrease is associated with Alfvén lines and is caused by the fast mode. 
region over which the wave modes will interact is small and can be neglected [e.g., Neubauer, 1980; Wright and Southwood, 1987]. For the Io torus parameter regime the extent of regions simultaneously affected by different wave modes is likely to be smaller than in the simulations presented here, because the Alfvén and sonic Mach numbers are probably farther apart (the Alfvén Mach number for the torus is certainly lower than the values used in the simulation, and the sonic Mach number may be higher). However, the dimension of the region generating the waves is relatively large (at least an Io diameter). Because the waves are all launched from the same rather extended region, the perturbations caused by the various modes should still overlap spatially close to Io, even if the Alfvén and sonic Mach numbers are very different.

The overlap of regions perturbed by the different wave modes can be important because the perturbations can affect each other. For example, Figure 12a shows that the rarefaction in plasma density, driven by the slow mode, changed the Alfvén speed significantly and thus affected intermediate and fast mode waves. However, in spite of the spatial overlap of the perturbed regions and the fact that the characteristic speeds in the simulation change as a result of the interaction, perturbations related to different MHD modes are reasonably well distinguished (at least qualitatively) based on the initial values of the characteristic speeds.

\section{Summary and Conclusions}

We have developed a time-dependent three-dimensional MHD simulation of plasma flow past Io. The spherical coordinate grid used in the simulation allows us to model the Io boundary more accurately and to have better resolution near Io than if a Cartesian grid were used. The simulation uses a two-step Lax-Wendroff method to solve the MHD equations. The poles of the spherical coordinate system $(\theta=0, \pi)$ present a difficulty because of singularities in the MHD equations. We have treated this problem in the simulations by deriving a new set of MHD equations, valid only at the poles. Io and its ionosphere are represented as a sphere of finite conductivity.

Our simulations show the formation of an Alfvén wing, a fundamental result expected from analytic theory. The total magnetic field bends in the vicinity of the Alfvén wing, but the Alfvén wing is most readily identified by examining the region where field-aligned currents are generated. The plasma flow above and below Io diverts around the Alfvén wing. An "Alfvén ellipse", the intersection of a cylindrical Alfvén wing with a given plane above Io, gives a reasonably good description of the obstacle presented by the Alfvén wing; however, the Alfvén wing is not an impenetrable obstacle. The flow in the Alfvén wing is reduced, but because Io has a finite conductivity, the flow is nonzero. In accordance with equation (1), the reduction of the flow electric field in the Alfven wing is greater for the simulations with higher $M_{A}$ and higher $\Sigma_{\text {Io. }}$.

In addition to the Alfvén wing, our simulations show perturbations associated with the other MHD modes. Most important is the slow mode. As previously reported by Linker et al. [1988] (and also described by Wright and Schwart $z$ [1990]), a standing slow mode wave attached to Io (with a rarefaction in pressure) forms.
The wave is qualitatively consistent with the slow mode propagating nearly parallel to $\vec{B}$. A smaller, plasma pressure enhancing slow mode wave occurs just downstream of Io. Perturbations in the total (plasma + magnetic) pressure are also manifested and indicate that fast mode waves are present. The total pressure signature is consistent with fast mode waves propagating nearly parallel to $\vec{B}$. The perturbations caused by all three MHD modes spatially overlap close to Io, and the Alfvén and sound speeds are themselves altered by the interaction. Despite these nonlinear effects, descriptions of the interaction based on the initial values of the characteristic velocities are qualitatively correct.

With the Galileo spacecraft en route to Jupiter, it is appropriate that we comment on the possibility of measuring the perturbations to the flow described here. While some caution must be used in direct predictions (the simulations are idealized and do not exactly match the Io torus parameter regime), we believe that many of the basic features we describe, such as the standing Alfvén and slow mode perturbations, will be present (albeit their exact positions will depend on the ambient value of the Alfvén and sonic Mach numbers). However, the Galileo spacecraft will pass through Io's wake and will not be in an optimum trajectory for observing these perturbations because they occur outside Io's equatorial plane. While observation of the Alfvén wing by Galileo may be difficult, its existence is not in doubt, as it was indirectly observed by Voyager 1 [Acuna et al., 1981]. The decrease in magnetic field strength downstream of Io (Figure 9a) should be observable by Galileo. However, the slow mode rarefaction wave may be observable only if $M_{S}$ is larger than considered here (of the order of 2.5-3), so that the perturbation intersects some portion of Io's geometrical wake. Observability will depend to some extent on the exact orbit parameters and the position of Io relative to the magnetic equatorial plane at the time of encounter. Trajectories of the proposed Jupiter Polar Orbiter mission [Siscoe et al., 1985] would allow a spacecraft to directly observe both the Alfven wing and the slow mode perturbations.

In this paper we have described results for simulations that did not include the effects of neutrals on the flow. Ionization of neutrals near Io (which has been examined previously in analytic papers by Goertz [1980] and Southwood and Dunlop [1984]) can have important effects in Io's wake (and thus be easily observable by Galileo). Linker et al. [1989] showed some of the effects that ionization can have on the plasma temperature near Io. Further simulation results for cases involving ionization will be shown in a forthcoming paper.

Acknowledgments. This work was supported by the Jet Propulsion Laboratory under contract 955232 , by NASA under grant NAGW-2506, by the California Space Institute under grant CS-21-90, and by the San Diego Supercomputer Center. One of us (J. Linker) would like to thank Tatsuke Ogino and Stephen Brecht for useful discussions that aided development of the simulation code.

The editor thanks two referees for their assistance in evaluating this paper.

\section{REFERENCES}

Acuna, M. H., F. M. Neubauer, and N. F. Ness, Standing Alfvén wave current system at Io: Voyager 1 observations, J. Geophys. Res., 86, 8513, 1981. 
Bagenal, F., Plasma conditions inside Io's orbit: Voyager measurements, J. Geophys. Res., 90, 311, 1985.

Bagenal, F., and J. D. Sullivan, Direct plasma measurements in the lo torus and inner magnetosphere of Jupiter, $J$. Geophys. Res., 86, 8447, 1981.

Barnett, A., In situ measurements of the plasma bulk velocity near the Io flux tube, J. Geophys. Res., 91, 3011, 1986.

Bigg, E. K., Influence of the satellite Io on Jupiter's decametric emission, Nature, 203, 1008, 1964.

Bohachevsky, I. O., and R. E. Mates, A direct method for calculation of the flow about an axisymmetric body at an angle of attack, $A I A A J ., 4,776,1966$.

Brecht, S. H., J. G. Lyon, J. A. Fedder, and K. Hain, A time dependent three dimensional simulation of the Earth's magnetosphere: Reconnection events, J. Geophys. Res., $87,6098,1982$.

Broadfoot, A. L., et al., Extreme ultraviolet observations from Voyager 1 encounter with Jupiter, Science, 204, 979, 1979.

Brown, R. A., Optical line emission from Io, in Exploration of the Planetary System, edited by A. Woszczyk and C. Iwaniszewska, p. 527, D. Reidel, Hingham, Mass., 1974.

Brown, R. A., and W. H. Ip, Atomic clouds as distributed sources for the Io plasma torus, Science, 219, 1493, 1981.

Drell, S. D., H. M. Foley, and M. A. Ruderman, Drag and propulsion of large satellites in the ionosphere: An Alfvén propulsion engine in space, J. Geophys. Res., 70, 3131, 1965.

Durrance, S. T., P. D. Feldman, and H. A. Weaver, Rocket detection of ultraviolet emission from neutral oxygen and sulfur in the Io torus, Astrophys. J., 267, L125, 1983.

Fedder, J. A., and J. G. Lyon, The solar wind-magnetosphere-ionosphere current voltage relationship, Geophys. Res. Lett., 14, 880, 1987.

Goertz, C. K., Io's interaction with the plasma torus, $J$. Geophys. Res., 85, 2949, 1980.

Goertz, C. K., and P. A. Deift, Io's interaction with the magnetosphere, Planet. Space Sci., 21, 1399, 1973.

Goldreich, P., and D. Lynden-Bell, Io, a Jovian unipolar inductor, Astrophys. J., 156, 59, 1969.

Hill, T. W., A. J. Dessler, and C. K. Goertz, Magnetospheric models, in Physics of the Jovian Magnetosphere, edited by A. Dessler, 353, Cambridge University Press, New York, 1983.

Kivelson, M. G., J. A. Slavin, and D. J. Southwood, Magnetospheres of the Galilean satellites, Science, 205, 491 1979.

Kliore, A., D. L. Cain, G. Fjeldbo, B. L. Seidel, and S. I. Rasool, Preliminary results on the atmospheres of Io and Jupiter from the Pioneer $10 \mathrm{~S}$-band occultation experiment, Science, 183, 323, 1974.

Kupo, I., Yu. Mekler, and A. Eviatar, Detection of ionized sulfur in the Jovian magnetosphere, Astrophys. J. Lett., $205,151,1976$.

Leboeuf, J. N. T. Tajima, C. F. Kennel, and J. M. Dawson, Global simulation of the three dimensional magnetosphere, Geophys. Res. Lett., 8, 257, 1981.

Linker, J. A., M. G. Kivelson, and R. J. Walker, An MHD simulation of plasma flow past Io: Alfvén and slow mode perturbations, Geophys. Res. Lett., 15, 1311, 1988.

Linker, J. A., M. G. Kivelson, and R. J. Walker, The effect of mass loading on the temperature of a flowing plasma, Geophys. Res. Lett., 16, 763, 1989.

Marshall, L., and W. F. Libby, Stimulation of Jupiter's radio emission by Io, Nature, 214, 126, 1967.

Mekler, Yu., and A. Eviatar, Spectroscopic observations of Io, Astrophys. J. Lett., 199, L151, 1974.

Ness, N. F., M. H. Acuna, R. P. Lepping, L. F. Burlaga, K. W. Behannon, and F. M. Neubauer, Magnetic field studies at Jupiter by Voyager 1: Preliminary results, Science, 209, 42, 1979 .

Neubauer, F. M., Possible strengths of dynamo magnetic fields of the Galilean satellites and of Titan, Geophys. Res. Lett., 5, 905, 1978.

Neubauer, F. M., Nonlinear standing Alfven wave current system at Io: Theory, J. Geophys. Res., 85, 1171, 1980.

Ogino, T., A three-dimensional MHD simulation of the interaction of the solar wind with the Earth's magnetosphere: The generation of field-aligned currents, $J$. Geophys. Res., 91, 6791, 1986.

Ogino, T., R. J. Walker, and M. Ashour-Abdalla, A three dimensional MHD simulation of the interaction of the solar wind with comet Halley, J. Geophys. Res., 93, 9568, 1988.

Pilcher, C. B., and D. F. Strobel, Emissions from neutrals and ions in the Jovian magnetosphere, in Satellites of Jupiter, edited by D. Morrison, p. 807, University of Arizona Press, Tucson, 1982.

Potter, D., Computational Physics, John Wiley, New York, 1973.

Richtmyer, R. D., and K. W. Morton, Difference Methods for Initial-Value Problems, Wiley Interscience, New York, 1967.

Schmidt, H. U., and R. Wegmann, Plasma flow and magnetic fields in comets, in Comets, edited by L.L. Wilkening, p. 538, University of Arizona Press, Tucson, 1982.

Siscoe, G. L., Solar system magnetohydrodynamics, in Solar Terrestrial Physics, edited by R. L. Carovillano and J. M. Forbes, p. 11, D. Reidel, Hingham, Mass., 1983.

Siscoe, G. L., R. A. Brown, A. F. Nagy, H. W. Moos, F. Scarf, and G. L. Tyler, Jupiter Polar Orbiter Workshop, Univ. of Calif. Los Angeles, 1985.

Southwood, D. J., and M. W. Dunlop, Mass pickup in subAlfvenic flow: A case study for Io, Planet. Space Sci., 92, 1079, 1984.

Southwood, D. J., M. G. Kivelson, R. J. Walker, and J. A. Slavin, Io and its plasma environment, J. Geophys. Res., $85,5959,1980$.

Strom, R. G., N. M. Schneider, R. J. Terrile, A. F. Cook, and C. Hansen, Volcanic eruptions on Io, J. Geophys. Res., $86,8593,1981$.

Trafton, L., T. Parkinson, and W. Macy, Jr., The spatial extent of sodium emission around Io, Astrophys. J. Lett., $190,185,1974$.

Wolf-Gladrow, F. M. Neubauer, and M. Lussem, Io's interaction with the plasma torus: A self-consistent model, $J$. Geophys. Res., 92, 9949, 1987.

Wright, A. N., Energetic particle absorption at Io, J. Geophys. Res., 92, 3155, 1987.

Wright, A. N., and S. J. Schwartz, The equilibrium of a conducting body embedded in a flowing plasma, J. Geophys. Res., 95, 4027, 1990.

Wright, A. N., and D. J. Southwood, Stationary Alfvenic structures, J. Geophys. Res., 92, 1167, 1987.

Wu, C. C., R. J. Walker, and J. M. Dawson, A three dimensional MHD model of the Earth's magnetosphere, Geophys. Res. Lett., 8, 523, 1981.

M.G. Kivelson and R. J. Walker, Institute of Geophysics and Planetary Physics, University of California, Los Angeles, CA 90024

J. A. Linker, Physics Department, University of California, Irvine, CA 92717.

(Received April 1, 1991; revised July 29, 1991; accepted July 29, 1991.) 